\title{
AN SDE APPROACH TO LEAFWISE DIFFUSIONS ON FOLIATED SPACES AND ITS APPLICATIONS
}

\author{
KIYOTAKA SUZAKI \\ (Received September 27, 2013, revised April 4, 2014)
}

\begin{abstract}
We construct leafwise diffusions on foliated spaces via SDE approach. The obtained diffusions are stochastically continuous and hence have the Feller property. Moreover our construction enables us to prove a central limit theorem for the leafwise diffusion on a compact foliated space in the same way as for a diffusion on a compact manifold.
\end{abstract}

1. Introduction. The ergodic theory of dynamical systems with invariant measures is well-studied and has been supplying many interesting results to various branches of mathematics. Foliations, and consequently foliated spaces are regarded as generalization of dynamical systems. For example, a nonsingular flow on a manifold corresponds to a foliation with one-dimensional leaves. Therefore if we can find a class of measures which inherits dynamical properties of foliated spaces, we can naturally extend the ergodic theory of dynamical systems to that of foliated spaces. In 1983, Garnett [5] considered a stochastic process along the leaves on a compact foliated Riemannian manifold, which is called a leafwise Brownian motion. She called the invariant measures for the leafwise Brownian motion harmonic measures and showed the existence of them. Moreover, she obtained some basic results in the ergodic theory; however, she could not prove the Feller property of the semi-group generated by the leafwise Brownian motion. It was Candel who proved this property in his paper [1]. He used a method for solving evolution equations and the Hille-Yosida theorem to construct a Feller semi-group generated by a leafwise elliptic differential operator on a foliated space. A diffusion process along the leaves was also constructed by using this semi-group. We call such a process a leafwise diffusion process (leafwise diffusion for short). The basic facts for foliated spaces, leafwise diffusions and harmonic measures are available in [2], [3] and [17].

Our purpose is to construct leafwise diffusions on a compact foliated space by an alternative approach. First we introduce stochastic differential equations on the foliated space to obtain a class of diffusions. It is shown that each of the equations has a unique strong solution (Theorem 2.1). We have to note that the solution cannot be expected to have a regularity with respect to starting points as a solution of SDE on a manifold has. Since foliated spaces do not always have a manifold structure by definition, the solution has the tangential regularity but does not always have the transverse regularity with respect to starting points. But we can show that the stochastic continuity of the solution with respect to starting points (Theorem

2010 Mathematics Subject Classification. Primary 37C40; Secondary 60J60, 60 F05.

Key words and phrases. Foliated space, leafwise diffusion process, harmonic measure, central limit theorem. 
2.1). This is strong enough for establishing the Feller property. It should be noted that Kanai [7] discusses the transverse regularity of leafwise diffusions on a special foliated manifold.

Next we verify that for any second order leafwise elliptic differential operator without zero order term, there exists a leafwise smooth Riemannian metric such that the operator is expressed as the sum of a leafwise smooth vector field and the leafwise Laplace-Beltrami operator induced by the metric. Applying our results to a stochastic differential equation on the bundle of orthonormal frames of the foliated space, we obtain a leafwise diffusion generated by the operator. In particular, the leafwise diffusion is obtained as a map defined on the classical Wiener space. Thus our results are applicable to an analogue of the well-known Eells-Elworthy-Malliavin construction of a diffusion on a manifold, the details of which can be found in [6, Chapter V-4].

As another application we prove a central limit theorem for a class of additive functionals of the leafwise diffusion starting at almost every point with respect to any harmonic measure (Theorem 2.8). Moreover we apply the result to the case when there is only one harmonic measure (Theorem 4.4). Our construction of leafwise diffusions enables us to prove these limit theorems in the way used in [15], in which limit theorems for a diffusion on a compact manifold were proved. We also note that the Feller property and the limit theorems are obtained in [14] more easily than in the present paper when the underlying leafwise diffusion is the leafwise Brownian motion on a mapping torus.

2. Preliminaries and main results. First of all we introduce some notation and basic facts. Let $W_{1}, W_{2}$ be topological spaces and $U$ an open set of $\mathbb{R}^{d} \times W_{1}$. Let $k$ be a nonnegative integer. A function $f: U \rightarrow \mathbb{R}$ is said to be of class $C_{L}^{k}$ on $U$ if $f(\cdot, z)$ is of $C^{k}$ for any $z$ and

$$
U \ni(y, z) \mapsto \frac{\partial^{i_{1}+i_{2}+\cdots+i_{d}}}{\partial^{i_{1}} y^{1} \cdots \partial^{i_{d}} y^{d}} f(y, z) \in \mathbb{R}
$$

is continuous for any multi-index $\left(i_{1}, i_{2}, \ldots, i_{d}\right)$ with $i_{1}+i_{2}+\cdots+i_{d} \leq k$. A map $f: U \rightarrow$ $\mathbb{R}^{p}$ is said to be of class $C_{L}^{k}$ if each of the component functions is of class $C_{L}^{k}$ on $U$. Let $V$ be an open set of $\mathbb{R}^{p} \times W_{2}$. A map $f: U \rightarrow V$ is said to be of class $C_{L}^{k}$ if it is locally of the form $f(y, z)=\left(f_{1}(y, z), f_{2}(z)\right)$, where $f_{1}$ is of class $C_{L}^{k}$ and $f_{2}$ is continuous. In this paper we call a map $f: U \rightarrow V$ a leafwise smooth map if it is of class $C_{L}^{k}$ for any nonnegative integer $k$.

Let $M, Z$ be locally compact, separable, metrizable spaces. $M$ is a $d$-dimensional foliated space (modeled transversely on $Z$ ) if there exist an open cover $\mathcal{U}=\left\{U_{\alpha}\right\}$ of $M$ and homeomorphisms $\left\{\varphi_{\alpha}: U_{\alpha} \rightarrow B_{\alpha, 1} \times B_{\alpha, 2}\right\}$ such that if $U_{\alpha} \cap U_{\beta} \neq \emptyset$, then $\varphi_{\beta} \circ \varphi_{\alpha}^{-1}$ : $\varphi_{\alpha}\left(U_{\alpha} \cap U_{\beta}\right) \rightarrow \varphi_{\beta}\left(U_{\alpha} \cap U_{\beta}\right)$ is leafwise smooth, where $B_{\alpha, 1}$ and $B_{\alpha, 2}$ are open sets of $\mathbb{R}^{d}$ and $Z$, respectively. Such a pair $\left(U_{\alpha}, \varphi_{\alpha}\right)$ is called a foliated chart and $\mathcal{U}$ is called a foliated atlas. For convenience we sometimes write $\left(y_{\alpha}, z_{\alpha}\right)$ instead of $\varphi_{\alpha}$. A plaque is a set of the form $\varphi_{\alpha}^{-1}\left(B_{\alpha, 1} \times\{z\}\right)$. We may assume that $\mathcal{U}$ is regular:

(1) For each $\alpha, \overline{U_{\alpha}}$ is a compact subset of a foliated chart $\left(W_{\alpha}, \psi_{\alpha}\right)$ and $\varphi_{\alpha}=\left.\psi_{\alpha}\right|_{U_{\alpha}}$. Hence we can consider the plaques of $\overline{U_{\alpha}}$. 
(2) $\mathcal{U}$ is locally finite.

(3) Given foliated charts $\left(U_{\alpha}, \varphi_{\alpha}\right),\left(U_{\beta}, \varphi_{\beta}\right) \in \mathcal{U}$ and a plaque $P \subset U_{\alpha}$, then $P$ meets at most one plaque of $\overline{U_{\beta}}$.

For any $x \in M$, we put

$$
\begin{aligned}
L_{x}=\{y \in M: & \text { there exist plaques } P_{1}, P_{2}, \ldots, P_{n} \\
& \text { such that } \left.x \in P_{1}, y \in P_{n} \text { and } P_{i} \cap P_{i+1} \neq \emptyset \text { for } 1 \leq i \leq n-1\right\} .
\end{aligned}
$$

The subset $L_{x}$ of $M$ is called the leaf passing through $x \in M$. $M$ is decomposed into the leaves $\mathcal{L}=\left\{L_{\lambda}\right\}_{\lambda \in \Lambda}$. One can easily see that each of the leaves is a $d$-dimensional smooth manifold. References for these fundamentals are found in [2, Chapter 11] and [13, Chapter II].

Next we introduce function spaces and tensor fields on $M$ in the same way as in the case of manifolds in [6, Chapter V]. In what follows, we assume that $M$ is compact. Let $C(M)$ be the Banach space of continuous functions on $M$ endowed with the supremum norm $\|\cdot\|_{\infty}$. Given a nonnegative integer $k$, we denote by $C_{L}^{k}(M)$ the totality of functions $f$ satisfying that $f \circ \varphi^{-1}: U \rightarrow \mathbb{R}$ is of class $C_{L}^{k}$ for any foliated chart $(U, \varphi)$. Note that $C_{L}^{0}(M)=C(M)$. Let $C_{L}^{\infty}(M)$ be the intersection $\bigcap_{k \geq 0} C_{L}^{k}(M)$. A function on $M$ is called a leafwise smooth function if it belongs to $C_{L}^{\infty}(M)$. One can easily construct a leafwise smooth function separating given two points in $M$ and hence $C_{L}^{\infty}(M)$ is dense in $C(M)$ by the StoneWeierstrass theorem.

In the following, we use the Einstein summation convention, i.e., the summation sign is omitted for repeated indices appearing once at the top and once at the bottom. For any point $x \in M$, we denote by $T_{x}(\mathcal{L})_{q}^{p}$ the tensor product

$$
\underbrace{T_{x}\left(L_{x}\right) \otimes T_{x}\left(L_{x}\right) \otimes \cdots \otimes T_{x}\left(L_{x}\right)}_{p} \otimes \underbrace{T_{x}\left(L_{x}\right)^{*} \otimes T_{x}\left(L_{x}\right)^{*} \otimes \cdots \otimes T_{x}\left(L_{x}\right)^{*}}_{q} .
$$

A foliated chart $(U,(y, z))$ containing $x$ naturally induces a basis

$$
\begin{array}{r}
\left\{\left(\frac{\partial}{\partial y^{i_{1}}}\right)_{x} \otimes\left(\frac{\partial}{\partial y^{i_{2}}}\right)_{x} \otimes \cdots \otimes\left(\frac{\partial}{\partial y^{i_{p}}}\right)_{x} \otimes\left(d y^{j_{1}}\right)_{x} \otimes\left(d y^{j_{2}}\right)_{x} \otimes \cdots \otimes\left(d y^{j_{q}}\right)_{x}\right. \\
\left.: i_{1}, i_{2}, \ldots, i_{p}, j_{1}, j_{2}, \ldots, j_{q}=1,2, \ldots, d\right\}
\end{array}
$$

of $T_{x}(\mathcal{L})_{q}^{p}$. A leafwise smooth $(p, q)$-tensor field on $M$ is a map $u: M \ni x \mapsto u(x) \in$ $T_{x}(\mathcal{L})_{q}^{p}$ whose components $\left\{u_{j_{1}, j_{2}, \ldots, j_{q}}^{i_{1}, i_{2}, \ldots, i_{p}}(y, z)\right\}$ with respect to the basis (2.1) are leafwise smooth in every foliated chart. The family $\left\{u_{j_{1}, j_{2}, \ldots, j_{q}}^{i_{1}, i_{2}, \ldots, i_{p}}(y, z)\right\}$ of components satisfies a rule under a change of coordinates $(y, z) \mapsto(\tilde{y}, \tilde{z})$ :

$$
\tilde{u}_{j_{1}, j_{2}, \ldots, j_{q}}^{i_{1}, i_{2}, \ldots, i_{p}}(\tilde{y}, \tilde{z})=\frac{\partial \tilde{y}^{i_{1}}}{\partial y^{k_{1}}} \frac{\partial \tilde{y}^{i_{2}}}{\partial y^{k_{2}}} \cdots \frac{\partial \tilde{y}^{i_{p}}}{\partial y^{k_{p}}} \frac{\partial y^{l_{1}}}{\partial \tilde{y}^{j_{1}}} \frac{\partial y^{l_{2}}}{\partial \tilde{y}^{j_{2}}} \cdots \frac{\partial y^{l_{q}}}{\partial \tilde{y}^{j_{q}}} u_{l_{1}, l_{2}, \ldots, l_{q}}^{k_{1}, k_{2}, \ldots, k_{p}}(y, z) .
$$


Conversely, if a family $\left\{u_{j_{1}, j_{2}, \ldots, j_{q}}^{i_{1}, i_{2}, \ldots, i_{p}}(y, z)\right\}$ of leafwise smooth functions is defined in every foliated chart and satisfies the rule (2.2), then there exists a unique leafwise smooth $(p, q)$ tensor field whose components coincide with it. By definition, if $L$ is a leaf of $M$, the restriction of $u$ to $L$ is a usual smooth $(p, q)$-tensor field on $L$. A leafwise smooth $(1,0)$-tensor field is called a leafwise smooth vector field. A leafwise smooth $(0,2)$-tensor field $g=\left\{g_{i j}(y, z)\right\}$ is called a leafwise smooth Riemannian metric on $M$ if the matrices $\left\{\left(g_{i j}(y, z)\right)\right\}$ are symmetric and positive definite. There exist many such tensor fields since every open cover of $M$ admits a subordinate leafwise smooth partition of unity (see [13, Proposition 2.8]). We introduce a leafwise smooth vector field on $M$ for later convenience. Given a leafwise smooth Rie-

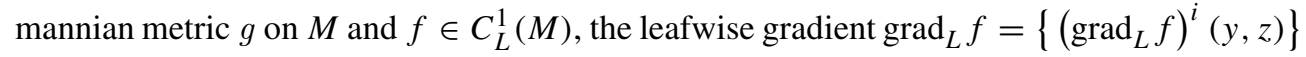
of $f$ is defined by

$$
\left(\operatorname{grad}_{L} f\right)^{i}(y, z)=g^{i j}(y, z) \frac{\partial f}{\partial y^{j}}(y, z)
$$

in each foliated chart $(U,(y, z))$. The length $\left\|\operatorname{grad}_{L} f(x)\right\|_{g}$ of $\operatorname{grad}_{L} f$ at $x$ is also defined by $\left\|\operatorname{grad}_{L} f(x)\right\|_{g}=\sqrt{g(x)\left(\operatorname{grad}_{L} f, \operatorname{grad}_{L} f\right)}$. The function $x \mapsto\left\|\operatorname{grad}_{L} f(x)\right\|_{g}$ is expressed as

$$
\left\|\operatorname{grad}_{L} f(y, z)\right\|_{g}=\sqrt{g^{i j}(y, z) \frac{\partial f}{\partial y^{i}}(y, z) \frac{\partial f}{\partial y^{j}}(y, z)}
$$

in each foliated chart $(U,(y, z))$.

Now we consider a stochastic differential equation on $M$. Given leafwise smooth vector fields $A_{0}, A_{1}, \ldots, A_{r}$ on $M$, we consider the following stochastic differential equation

$$
d X(t)=A_{\alpha}(X(t)) \circ d B^{\alpha}(t)+A_{0}(X(t)) d t .
$$

Let $\left(\Omega, \mathcal{F}, P,\left(\mathcal{F}_{t}\right)_{t \geq 0}\right)$ be a usual filtered probability space, i.e., $(\Omega, \mathcal{F}, P)$ is a complete probability space and $\left(\mathcal{F}_{t}\right)_{t \geq 0}$ is a right-continuous filtration such that $\mathcal{F}_{0}$ contains all $P$ null sets. For example, the classical $r$-dimensional Wiener space with canonical filtration $\left(W_{0}^{r}, \mathcal{F}^{W}, P^{W},\left(\mathcal{F}_{t}^{W}\right)_{t \geq 0}\right)$ is a usual filtered probability space, where $W_{0}^{r}$ is the totality of continuous maps $w:[0, \infty) \rightarrow \mathbb{R}^{r}$ with $w(0)=0$ endowed with the compact-open topology, $P^{W}$ is the $r$-dimensional Wiener measure, $\mathcal{F}^{W}$ is the completion of the topological Borel $\sigma$-field of $W_{0}^{r}$ by $P^{W}$ and $\mathcal{F}_{t}^{W}$ is the $\sigma$-field generated by the Borel cylinder sets up to time $t$ and all $P^{W}$-null sets. Let $W_{L}(M)$ be the totality of continuous maps $\omega:[0, \infty) \rightarrow M$ such that the image is contained in a single leaf. It is easy to see that $W_{L}(M)$ endowed with the compact-open topology is a complete, separable, metrizable space. We denote by $\mathcal{B}\left(W_{L}(M)\right)$ and $\mathcal{B}_{t}\left(W_{L}(M)\right)$ the topological Borel $\sigma$-field of $W_{L}(M)$ and the sub- $\sigma$-field of $\mathcal{B}\left(W_{L}(M)\right)$ generated by the Borel cylinder sets up to time $t$, respectively. The $\sigma$-fields $\mathcal{B}(M)$, $\mathcal{B}\left(W_{0}^{r}\right), \mathcal{B}_{t}\left(W_{0}^{r}\right)$ etc. are defined similarly. We say that an $\left(\mathcal{F}_{t}\right)$-adapted, $W_{L}(M)$-valued random variable $X=\{X(t)\}_{t \geq 0}$ on $\left(\Omega, \mathcal{F}, P,\left(\mathcal{F}_{t}\right)_{t \geq 0}\right)$ is a solution of (2.5) if there exists an 
$r$-dimensional $\left(\mathcal{F}_{t}\right)$-Brownian motion $B=\{B(t)\}_{t \geq 0}$ with $B(0)=0$ such that

$$
f(X(t))-f(X(0))=\int_{0}^{t}\left(A_{\alpha} f\right)(X(s)) \circ d B^{\alpha}(s)+\int_{0}^{t}\left(A_{0} f\right)(X(s)) d s \quad P \text {-a.s. }
$$

for any $f \in C_{L}^{2}(M)$, where the first term on the right-hand side is understood in the sense of the Fisk-Stratonovich integral (see [6, Chapter III-1] ).

For any topological space $S$ and any Borel probability measure $\mu$ on $S$, we put

$$
\begin{aligned}
\mathcal{F}(S)^{\mu} & =\overline{\left(\mathcal{B}(S) \otimes \mathcal{B}\left(W_{0}^{r}\right)\right)} \mu \otimes P^{W}=\text { the completion of } \mathcal{B}(S) \otimes \mathcal{B}\left(W_{0}^{r}\right) \text { by } \mu \otimes P^{W}, \\
\mathcal{F}_{t}(S)^{\mu} & =\left(\mathcal{B}(S) \otimes \mathcal{B}_{t}\left(W_{0}^{r}\right)\right) \vee \mathcal{N}\left(\mu \otimes P^{W}\right) \\
& =\text { the } \sigma \text {-field generated by } \mathcal{B}(S) \otimes \mathcal{B}_{t}\left(W_{0}^{r}\right) \text { and all } \mu \otimes P^{W} \text {-null sets, }
\end{aligned}
$$

and

$$
\widehat{\mathcal{F}}(S)=\bigcap_{\mu: \text { Borel probability measure on } M} \mathcal{F}(S)^{\mu}, \widehat{\mathcal{F}}_{t}(S)=\bigcap_{\mu: \text { Borel probability measure on } M} \mathcal{F}_{t}(S)^{\mu} .
$$

In the next section we will construct solutions $\left\{X^{x}\right\}_{x \in M}$ of (2.5) on the Wiener space $\left(W_{0}^{r}, \mathcal{F}^{W}, P^{W},\left(\mathcal{F}_{t}^{W}\right)_{t \geq 0}\right)$ such that $X^{x}(0)=x P^{W}$-a.s. for each $x \in M$. Furthermore, we will see that the family $\left\{X^{x}\right\}_{x \in M}$ of stochastic processes is stochastically continuous with respect to $x$. Precisely we will obtain the following.

THEOREM 2.1. There exists a map $F: M \times W_{0}^{r} \rightarrow W_{L}(M)$ satisfying the following:

(1) $F$ is $\widehat{\mathcal{F}}(M) / \mathcal{B}\left(W_{L}(M)\right)$-measurable.

(2) $F$ is $\widehat{\mathcal{F}}_{t}(M) / \mathcal{B}_{t}\left(W_{L}(M)\right)$-measurable for any $t \geq 0$.

(3) Suppose that an $\left(\mathcal{F}_{t}\right)$-adapted, $W_{L}(M)$-valued random variable $X=\{X(t)\}_{t \geq 0}$ is a solution of (2.5) with an $\left(\mathcal{F}_{t}\right)$-Brownian motion $B=\left\{B_{t}\right\}_{t \geq 0}$ on a usual filtered probability space $\left(\Omega, \mathcal{F}, P,\left(\mathcal{F}_{t}\right)_{t \geq 0}\right)$. Then $X=F(X(0), B) P$-a.s.

(4) If $B=\{B(t)\}_{t \geq 0}$ is an $\left(\mathcal{F}_{t}\right)$-Brownian motion and $\xi$ is an $M$-valued $\mathcal{F}_{0}$-random variable on $\left(\Omega, \mathcal{F}, P,\left(\mathcal{F}_{t}\right)_{t \geq 0}\right)$, then $F(\xi, B)$ is a solution of $(2.5)$ on $(\Omega, \mathcal{F}, P$, $\left.\left(\mathcal{F}_{t}\right)_{t \geq 0}\right)$ with the initial value $\xi$.

Therefore, if we define a map $X^{x}: W_{0}^{r} \rightarrow W_{L}(M)$ for a fixed $x \in M$ by $X^{x}(t, w)=$ $F(x, w)(t)$ for $(t, w) \in[0, \infty) \times W_{0}^{r}$, then the stochastic process $X^{x}=\left\{X^{x}(t)\right\}_{t \geq 0}$ is a solution of (2.5) with the Brownian motion $w=\{w(t)\}_{t \geq 0}$ and the initial distribution $\delta_{x}$ on $\left(W_{0}^{r}, \mathcal{F}^{W}, P^{W},\left(\mathcal{F}_{t}^{W}\right)_{t \geq 0}\right)$. Furthermore, if $d_{M}$ is a metric on $M$, then for any $\varepsilon>0$ and $T>0$, there exists $\delta>0$ such that

$$
P^{W}\left(\sup _{0 \leq t \leq T} d_{M}\left(X^{x}(t), X^{\tilde{x}}(t)\right)<\varepsilon\right) \geq 1-\varepsilon
$$

for any $x, \tilde{x} \in M$ with $d_{M}(x, \tilde{x})<\delta$.

The proof of Theorem 2.1 will be given in the next section.

REMARK 2.2. (1) We sometimes write $X(t, x, w)$ instead of $X^{x}(t, w)$ for convenience. The pathewise uniqueness (the assertion (3) in the above) yields that if $\sigma$ is a 
bounded $\left(\mathcal{F}_{t}^{W}\right)$-stopping time, then $X(t+\sigma(w), x, w)=X\left(t, X(\sigma(w), x, w), \theta_{\sigma}(w)\right)$ for $t \geq 0, P^{W}$-a.s. $w$, where $\theta_{\sigma}: W_{0}^{r} \rightarrow W_{0}^{r}$ is defined by $\theta_{\sigma} w(\cdot)=w(\cdot+\sigma(w))-w(\sigma(w))$. In particular, the family of laws of the stochastic processes $\left\{X^{x}\right\}_{x \in M}$ is a strongly Markovian system.

(2) If $M$ has a smooth manifold structure and $A_{0}, A_{1}, \ldots, A_{r}$ are smooth vector fields on $M$, then $\left\{X^{x}\right\}_{x \in M}$ has a modification such that the map $x \mapsto X(t, x, w)$ is smooth $P^{W}$-a.s.w for any $t \geq 0$ (see [6, Theorem V-2.4]). Therefore we easily see that the family $\left\{X^{x}\right\}_{x \in M}$ has the Feller property. As seen from the Corollary below, we need the latter part of Theorem 2.1 to show that the family $\left\{X^{x}\right\}_{x \in M}$ has the Feller property in the general case.

We put

$$
T(t) f(x)=E\left[f\left(X^{x}(t)\right)\right]
$$

for any bounded Borel measurable function $f$ on $M$ and $t \geq 0$. From Theorem 2.1, we see that the family of positive operators $\{T(t)\}_{t \geq 0}$ turns out to be a Feller semi-group on $C(M)$ with a closed extension of $A=(1 / 2) \sum_{\alpha} A_{\alpha} A_{\alpha}+A_{0}$ as the infinitesimal generator.

COROLLARY 2.3. We have the following:

(1) For any $t \geq 0$ and $f \in C(M),\|T(t) f\|_{\infty} \leq\|f\|_{\infty}$.

(2) For any $t \geq 0$ we have $T(t) C(M) \subset C(M)$.

(3) For $t, s \geq 0$ and $f \in C(M)$, we have $T(t+s) f=T(t) T(s) f$.

(4) For any $f \in C(M), \lim _{t \downarrow}\|T(t) f-f\|_{\infty}=0$.

(5) The infinitesimal generator of the semi-group $\{T(t)\}_{t \geq 0}$ is an extension of $A$ and the domain contains $C_{L}^{2}(M)$.

Proof. The assertion (1) is obvious from (2.7). Take any $f \in C(M)$ and $x \in M$. For any $\varepsilon>0$, we can choose $\delta>0$ such that $|f(x)-f(\tilde{x})|<\varepsilon$ whenever $d_{M}(x, \tilde{x})<\delta$. Thus we have

$$
\begin{aligned}
|T(t) f(x)-T(t) f(\tilde{x})| \leq & E\left[\left|f\left(X^{x}(t)\right)-f\left(X^{\tilde{x}}(t)\right)\right|\right] \\
\leq & E\left[\mid f\left(X^{x}(t)-f\left(X^{\tilde{x}}(t)\right) \mid: d_{M}\left(X^{x}(t), X^{\tilde{x}}(t)\right)<\delta\right]\right. \\
& +E\left[\left|f\left(X^{x}(t)\right)-f\left(X^{\tilde{x}}(t)\right)\right|: d_{M}\left(X^{x}(t), X^{\tilde{x}}(t)\right) \geq \delta\right] \\
< & \varepsilon+2\|f\|_{\infty} P^{W}\left(d_{M}\left(X^{x}(t), X^{\tilde{x}}(t)\right) \geq \delta\right) \rightarrow \varepsilon \quad(\tilde{x} \rightarrow x)
\end{aligned}
$$

by Theorem 2.1. This implies that the assertion (2) is valid. The assertion (3) follows from the Markov property of the stochastic processes $\left\{X^{x}\right\}_{x \in M}$. To verify the assertion (4) it suffices to show that $\lim _{t \downarrow 0}\|T(t) f-f\|_{\infty}=0$ for any $f \in C_{L}^{2}(M)$. Since $X^{x}=\left\{X^{x}(t)\right\}_{t \geq 0}$ is a solution of (2.5) with the initial distribution $\delta_{x}$, we have

$$
f\left(X^{x}(t)\right)-f(x)=\text { a martingale with mean } 0+\int_{0}^{t} A f\left(X^{x}(s)\right) d s .
$$

By taking expectation and supremum norms in the both sides we obtain

$$
\|T(t) f-f\|_{\infty} \leq\left\|E\left[\int_{0}^{t} A f\left(X^{x}(s)\right) d s\right]\right\|_{\infty} \leq\|A f\|_{\infty} t \rightarrow 0 \quad(t \rightarrow 0) .
$$


Thus the assertion (4) is valid. It remains to prove the assertion (5). We have to show that

$$
\lim _{t \rightarrow 0}\left\|\frac{T(t) f-f}{t}-A f\right\|_{\infty}=0
$$

for any $f \in C_{L}^{2}(M)$. From the equation (2.8) and the Fubini theorem we have

Therefore we have

$$
T(t) f(x)-f(x)=\int_{0}^{t} T(s) A f(x) d s .
$$

$$
\left\|\frac{T(t) f-f}{t}-A f\right\|_{\infty} \leq \frac{1}{t} \int_{0}^{t}\|T(s) A f-A f\|_{\infty} d s .
$$

Applying the assertion (4) to $A f$, we obtain the desired result.

Now we construct a diffusion process generated by a leafwise ellptic differential operator with assuming the validity of Theorem 2.1 . We say that a linear operator $A: C_{L}^{2}(M) \rightarrow$ $C(M)$ is a second order leafwise elliptic differential operator (without zero order term) on $M$ if it is expressed as

$$
A f(y, z)=\frac{1}{2} \alpha^{i j}(y, z) \frac{\partial^{2}}{\partial y^{i} \partial y^{j}} f(y, z)+\beta^{i}(y, z) \frac{\partial}{\partial y^{i}} f(y, z)
$$

for $f \in C_{L}^{2}(M)$ in each foliated chart $(U,(y, z))$, where the coefficients $\alpha^{i j}, \beta^{i}, i, j=$ $1,2, \ldots, d$ are leafwise smooth and the matrices $\left\{\left(\alpha^{i j}(y, z)\right)\right\}$ are symmetric, positive definite. The function $A f$ is also defined by the local expression if $f$ is of class $C^{2}$ along each leaf. The family $\left\{\alpha^{i j}(y, z)\right\}$ of leafwise smooth functions defines a $(2,0)$-tensor field on $M$ and hence we see that the inverse matrices $\left\{\left(g_{i j}(y, z)\right)\right\}$ of $\left\{\left(\alpha^{i j}(y, z)\right)\right\}$ defines a leafwise smooth Riemannian metric $g=\left\{g_{i j}(y, z)\right\}$ on $M$. Let $\left\{\left(\Gamma_{g}\right)_{i j}^{k}(y, z)\right\}$ be the leafwise Levi-Civita connection and $\Delta_{g}$ the leafwise Laplace-Beltrami operator induced by $g$ on $M$. That is, in any foliated chart $(U,(y, z))$,

$$
\left(\Gamma_{g}\right)_{i j}^{k}(y, z)=\frac{1}{2}\left(\frac{\partial}{\partial y^{i}} g_{m j}(y, z)+\frac{\partial}{\partial y^{j}} g_{i m}(y, z)-\frac{\partial}{\partial y^{m}} g_{i j}(y, z)\right) g^{k m}(y, z),
$$

where $\left(g^{i j}(y, z)\right)=\left(g_{i j}(y, z)\right)^{-1}$, and

$$
\Delta_{g} f(y, z)=g^{i j}(y, z) \frac{\partial^{2}}{\partial y^{i} \partial y^{j}} f(y, z)-g^{i j}(y, z)\left(\Gamma_{g}\right)_{i j}^{k}(y, z) \frac{\partial}{\partial y^{k}} f(y, z) .
$$

We define leafwise smooth functions $\left\{b^{i}(y, z)\right\}$ in every foliated chart by

$$
b^{i}(y, z)=\beta^{i}(y, z)+\frac{1}{2} g^{j k}(y, z)\left(\Gamma_{g}\right)_{j k}^{i}(y, z) .
$$

Then we see that $b=\left\{b^{i}(y, z)\right\}$ is a leafwise smooth vector field and

$$
A=\frac{1}{2} \Delta_{g}+b
$$

Therefore any second order leafwise smooth elliptic differential operator can be expressed as the form (2.9). 
We consider sets

$$
G L(\mathcal{L})=\left\{r=(x, e): e \text { is a base of } T_{x}(\mathcal{L})_{0}^{1}\right\}
$$

and

$$
O(\mathcal{L})=\left\{r=(x, e): e \text { is an orthonormal base of } T_{x}(\mathcal{L})_{0}^{1} \text { with respect to } g\right\} .
$$

For $\lambda \in \Lambda$, let $G L\left(L_{\lambda}\right)$ be the bundle of linear frames and $O\left(L_{\lambda}\right)$ the bundle of orthonormal frames on $\left(L_{\lambda},\left.g\right|_{L_{\lambda}}\right)$. We can easily show that $G L(\mathcal{L})$ and $O(\mathcal{L})$ are foliated spaces with $\left\{G L\left(L_{\lambda}\right)\right\}_{\lambda \in \Lambda}$ and $\left\{O\left(L_{\lambda}\right)\right\}_{\lambda \in \Lambda}$ as the leaves, respectively. Indeed, a foliated atlas $\mathcal{U}=$ $\left\{\left(U_{\alpha}, \varphi_{\alpha}\right)\right\}$ of $M$ gives a foliated atlas of $G L(\mathcal{L})$ as follows. For each $\alpha$, we define a set $\widetilde{U}_{\alpha}$ by

$$
\widetilde{U}_{\alpha}=\left\{r=(x, e) \in G L(\mathcal{L}): x \in U_{\alpha}\right\}
$$

and a map $\tilde{\varphi}$ from $\widetilde{U}$ onto $\varphi_{\alpha}\left(U_{\alpha}\right) \times G L(d, \mathbb{R}) \subset \mathbb{R}^{d} \times Z \times \mathbb{R}^{d^{2}}$ by

$$
\tilde{\varphi}_{\alpha}(r)=\tilde{\varphi}_{\alpha}(x, e)=\left(\varphi_{\alpha}(x),\left(e_{j}^{i}, i, j=1,2, \ldots, d\right)\right),
$$

where $e=\left(e_{1}, e_{2}, \ldots, e_{d}\right)$ and

$$
e_{j}=e_{j}^{i}\left(\frac{\partial}{\partial y_{\alpha}^{i}}\right)_{x} \in T_{x}(\mathcal{L})_{0}^{1} .
$$

The pair $\left(\widetilde{U}_{\alpha}, \widetilde{\varphi}_{\alpha}\right)$ gives a foliated chart of $G L(\mathcal{L})$ and we see that $\tilde{\mathcal{U}}=\left\{\left(\widetilde{U}_{\alpha}, \widetilde{\varphi}_{\alpha}\right)\right\}$ is a foliated atlas of $G L(\mathcal{L})$. It is similarly verified for $O(\mathcal{L})$. In particular, if $M$ is compact then so is $O(\mathcal{L})$. An element $a$ of the real orthogonal group $O(d, \mathbb{R})$ acts on $O(\mathcal{L})$ from the right by $r \cdot a=(x, e a)$ for $r=(x, e)$, where $e a=\left((e a)_{1},(e a)_{2}, \ldots,(e a)_{d}\right)$ is an orthonormal base of $T_{x}(\mathcal{L})_{0}^{1}$ defined by $(e a)_{j}=a_{j}^{i} e_{i}, j=1,2, \ldots, d$. Thus $O(\mathcal{L})$ is a (leafwise smooth) principal fibre bundle with the structural group $O(d, \mathbb{R})$.

Leafwise smooth vector fields $\widetilde{H}_{0}, \widetilde{H}_{1}, \ldots, \widetilde{H}_{d}$ on $G L(\mathcal{L})$ are defined by

$$
\begin{aligned}
\widetilde{H}_{0} & =b^{i}(y, z) \frac{\partial}{\partial y^{i}}-\left(\Gamma_{g}\right)_{i j}^{q}(y, z) b^{i}(y, z) e_{p}^{j} \frac{\partial}{\partial e_{p}^{q}}, \\
\widetilde{H}_{\alpha} & =e_{\alpha}^{i} \frac{\partial}{\partial y^{i}}-\left(\Gamma_{g}\right)_{i j}^{q}(y, z) e_{\alpha}^{i} e_{p}^{j} \frac{\partial}{\partial e_{p}^{q}}, \quad \alpha=1,2, \ldots, d,
\end{aligned}
$$

in each foliated chart $\left(\widetilde{U},\left(y, z,\left(e_{j}^{i}\right)\right)\right)$, i.e., these vector fields are defined so that $\left.\widetilde{H}_{0}\right|_{G L(L)}$ is the horizontal lift of $\left.b\right|_{L}$ and $\left\{\left.\widetilde{H}_{1}\right|_{G L(L)},\left.\widetilde{H}_{2}\right|_{G L(L)}, \ldots,\left.\widetilde{H}_{d}\right|_{G L(L)}\right\}$ is the system of canonical horizontal vector fields on $G L(L)$ if $L$ is a leaf of $M$. We also denote the restrictions of these vector fields to $O(\mathcal{L})$ by the same symbols. Consider the stochastic differential equation on $O(\mathcal{L})$ given by

$$
d r(t)=\widetilde{H}_{\alpha}(r(t)) \circ d B^{\alpha}(t)+\widetilde{H}_{0}(r(t)) d t .
$$

Applying Theorem 2.1 to the equation (2.11), we obtain a solution $\{r(t, r)\}_{t \geq 0}$ of (2.11) with the initial distribution $\delta_{r}$ on the Wiener space $\left(W_{0}^{d}, \mathcal{F}^{W}, P^{W},\left(\mathcal{F}_{t}^{W}\right)_{t \geq 0}\right)$ for each $r \in O(\mathcal{L})$. Let $\pi: O(\mathcal{L}) \ni r=(x, e) \mapsto x \in M$ be the natural projection. We consider a stochastic 
process $X(r)=\{X(t, r)\}_{t \geq 0}$ defined by $X(t, r)=\pi(r(t, r))$. By the pathwise uniqueness of solutions for (2.11) we see that

$$
r(t, r, a w) \cdot a=r(t, r \cdot a, w)
$$

for any $t \geq 0, P^{W}$-a.s. $w$ and any $a \in O(d, \mathbb{R})$, where $a w=\{a w(t)\}_{t \geq 0}$ is another $d$ dimensional Brownian motion on $\left(W_{0}^{d}, \mathcal{F}^{W}, P^{W},\left(\mathcal{F}_{t}^{W}\right)_{t \geq 0}\right)$. So we have

$$
X(t, r, a w)=X(t, r \cdot a, w)
$$

for any $t \geq 0, P^{W}$-a.s. $w$ and any $a \in O(d, \mathbb{R})$. Therefore the law $P^{r}$ of $X(r)$ depends only on $x=\pi(r)$. For any $x \in M$ we take $r \in O(\mathcal{L})$ with $\pi(r)=x$ and put

$$
X(t, x)\left(\text { or } X^{x}(t)\right)=X(t, r) \text { for } t \geq 0 \quad \text { and } \quad P^{x}=P^{r} .
$$

Then $P^{x}$ is the law of $X^{x}$ and the family $\left\{P^{x}\right\}_{x \in M}$ is a strongly Markovian system. We can also prove that the family $\left\{X^{x}\right\}_{x \in M}$ gives a Feller semi-group with an extension of $A=$ $(1 / 2) \Delta_{g}+b$ as the infinitesimal generator. Precisely we have the following.

COROLlary 2.4. For any bounded Borel measurable function $f$ on $M$ and $t \geq 0$ let $T(t) f(x)=E\left[f\left(X^{x}(t)\right)\right]$. Then the assertions (1)-(5) of Corollary 2.3 are valid for $\{T(t)\}_{t \geq 0}$ and $A=(1 / 2) \Delta_{g}+b$.

Proof. Put $\widetilde{H}=(1 / 2) \sum_{\alpha} \widetilde{H}_{\alpha} \widetilde{H}_{\alpha}+\widetilde{H}_{0}$. We notice that $f \in C(M)$ implies $f \circ \pi \in$ $C(O(\mathcal{L}))$. Moreover $f \in C_{L}^{2}(M)$ implies $f \circ \pi \in C_{L}^{2}(O(\mathcal{L}))$ and $\widetilde{H}(f \circ \pi)=A f$. Applying

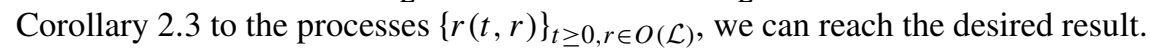

Now we summarize the above mentioned results as the following theorem.

THEOREM 2.5. Let $M$ be a compact foliated space, A a second order leafwise smooth elliptic differential operator defined by (2.9), $\widetilde{H}_{0}$ the horizontal lift of $b$ and $\left\{\widetilde{H}_{1}, \widetilde{H}_{2}, \ldots, \widetilde{H}_{d}\right\}$ the system of canonical horizontal vector fields (with respect to the leafwise Levi-Civita connection) defined by (2.10). Consider the stochastic differential equation (2.11) on $O(\mathcal{L})$. Solutions of the equation define the family $\{r(t, r)\}_{t \geq 0, r \in O(\mathcal{L})}$ of diffusion processes on $O(\mathcal{L})$, which is stochastically continuous with respect to $r$. Their projections $\left\{X^{x}\right\}_{x \in M}$ to $M$ give a Feller semi-group $\{T(t)\}_{t \geq 0}$ with an extension of $A$ as the infinitesimal generator.

REMARK 2.6. (1) The infinitesimal generator of $\{T(t)\}_{t \geq 0}$ and its domain are identified via the Hille-Yosida theorem in [1].

(2) By Corollary 2.4 and the Markov property of $\left\{P^{x}\right\}_{x \in M}$, we see that a function defined by $x \mapsto P^{x}(B)$ is $\mathcal{B}(M)$-measurable for any Borel measurable set $B$ of $W_{L}(M)$.

We call the stochastic process $X^{x}=\left\{X^{x}(t)\right\}_{t \geq 0}$ constructed in the above the $A$-leafwise diffusion process on $M$ starting at $x$. The family $X=\left\{X^{x}\right\}_{x \in M}$ of the $A$-leafwise diffusion processes is simply called the $A$-leafwise diffusion on $M$.

To state another one of the main results we consider $A$-harmonic measures. A Borel measure $m$ is called an $A$-harmonic measure if

$$
\int_{M} A f d m=0
$$


for any $f \in C(M)$ which is of class $C^{2}$ along each leaf and satisfies $A f \in C(M)$. The $A$-harmonic measure is characterized as an invariant measure for the $A$-leafwise diffusion, i.e.,

holds for any $f \in C(M)$ and for any $t \geq 0$ (see [1]).

$$
\int_{M} T(t) f d m=\int_{M} f d m
$$

As another application of our construction, we show a central limit theorem for a class of additive functionals. To this end we need the following result which is a consequence of the ergodic theorem and the martingale convergence theorem. We note that the following is an analogue of [14, Proposition 2.7].

PROPOSITION 2.7.

(1) Consider the set

$$
\begin{gathered}
Q_{X}=\left\{x \in M: \lim _{t \rightarrow \infty} \frac{1}{t} \int_{0}^{t} f\left(X^{x}(s)\right) d s=\lim _{t \rightarrow \infty} \frac{1}{t} \int_{0}^{t} T(s) f(x) d s\right. \\
\text { for any } \left.f \in C(M), P^{W} \text {-a.s. }\right\} \\
=\left\{x \in M: \lim _{t \rightarrow \infty} \frac{1}{t} \int_{0}^{t} f(\omega(s)) d s=\lim _{t \rightarrow \infty} \frac{1}{t} \int_{0}^{t} E^{\omega(0)}[f(\omega(s))] d s\right. \\
\text { for any } \left.f \in C(M), P^{x} \text {-a.s. } \omega\right\},
\end{gathered}
$$

where the symbol $E^{x}$ means taking the expectation with respect to $P^{x}$. Then $Q_{X}$ is Borel measurable subset of $M$.

(2) $m\left(Q_{X}\right)=1$ for any A-harmonic probability measure $m$.

(3) For any $x \in Q_{X}$, there exist an A-harmonic probability measure $m_{x}$ and a measurable set $\Lambda_{x}$ with $P^{W}\left(\Lambda_{x}\right)=1$ such that $w \in \Lambda_{x}$ yields

$$
\lim _{t \rightarrow \infty} \frac{1}{t} \int_{0}^{t} f\left(X^{x}(s, w)\right) d s=\int_{M} f d m_{x}
$$

for any $f \in C(M)$.

Proof. (1) Consider the set

$$
Q_{1}\left(W_{L}(M)\right)=\left\{\omega \in W_{L}(M): \lim _{t \rightarrow \infty} \frac{1}{t} \int_{0}^{t} f(\omega(s)) d s \text { exists for any } f \in C(M)\right\} .
$$

Obviously the set

$$
Q_{1}\left(W_{L}(M), f\right)=\left\{\omega \in W_{L}(M): \lim _{t \rightarrow \infty} \frac{1}{t} \int_{0}^{t} f(\omega(s)) d s \text { exists }\right\}
$$

is $\mathcal{B}\left(W_{L}(M)\right)$-measurable for each $f \in C(M)$. Let $\left\{f_{n}\right\}$ be a countable dense subset of $C(M)$. It is easy to see that

$$
Q_{1}\left(W_{L}(M)\right)=\bigcap_{n=1}^{\infty} Q_{1}\left(W_{L}(M), f_{n}\right)
$$


Therefore $Q_{1}\left(W_{L}(M)\right)$ is $\mathcal{B}\left(W_{L}(M)\right)$-measurable. For a fixed $f \in C(M)$, we define bounded functions on $W_{L}(M)$ by

$$
\operatorname{Avr}(f)(\omega)= \begin{cases}\lim _{t \rightarrow \infty} \frac{1}{t} \int_{0}^{t} f(\omega(s)) d s & \omega \in Q_{1}\left(W_{L}(M)\right) \\ 0 & \omega \notin Q_{1}\left(W_{L}(M)\right)\end{cases}
$$

and

$$
A v r_{0}(f)(\omega)=E^{\omega(0)}[A v r(f)]
$$

We can show that the set

$$
Q_{2}\left(W_{L}(M)\right)=\left\{\omega \in W_{L}(M): \operatorname{Avr}(f)(\omega)=A v r_{0}(f)(\omega) \text { for any } f \in C(M)\right\}
$$

is $\mathcal{B}\left(W_{L}(M)\right)$-measurable in the same way as $Q_{1}\left(W_{L}(M)\right)$. Since $x \mapsto P^{x}\left(Q_{1}\left(W_{L}(M)\right) \cap\right.$ $\left.Q_{2}\left(W_{L}(M)\right)\right)$ is a $\mathcal{B}(M)$-measurable map and

$$
Q_{X}=\left\{x \in M: P^{x}\left(Q_{1}\left(W_{L}(M)\right) \cap Q_{2}\left(W_{L}(M)\right)\right)=1\right\},
$$

the assertion (1) is valid.

(2) Take any $A$-harmonic probability measure $m$ and $f \in C(M)$. Let $P^{m}$ be a Borel probability measure on $W_{L}(M)$ defined by $P^{m}(d \omega)=P^{x}(d \omega) m(d x)$. In order to verify the assertion (2) it suffices to show that $P^{m}\left(Q_{1}\left(W_{L}(M)\right)\right)=1$ and $P^{m}\left(Q_{2}\left(W_{L}(M)\right)\right)=1$. We consider the semi-flow of translations $\left\{\sigma_{t}\right\}$ on $W_{L}(M)$ defined by

$$
\left(\sigma_{t} \omega\right)(s)=\omega(t+s) \quad \text { for } s \geq 0 .
$$

The diffusion invariance of $m$ implies that $\left(\left\{\sigma_{t}\right\}_{t \geq 0}, P^{m}\right)$ is a continuous parameter measurepreserving dynamical system. Applying the ergodic theorem, we have

$$
P^{m}\left(Q_{1}\left(W_{L}(M), f\right)\right)=1
$$

and hence $P^{m}\left(Q_{1}\left(W_{L}(M)\right)\right)=1$. Next we notice that $A v r(f) \circ \sigma_{t}=A v r(f)$ for any $t \geq 0$. The Markov property of $\left\{P^{x}\right\}_{x \in M}$ and the martingale convergence theorem yield that

$$
\begin{aligned}
A v r_{0}(f) \circ \sigma_{t}(\omega) & =E^{\omega(t)}[\operatorname{Avr}(f)]=E^{x}\left[\operatorname{Avr}(f) \circ \sigma_{t} \mid \mathcal{B}_{t}\left(W_{L}(M)\right)\right](\omega) \\
& =E^{x}\left[\operatorname{Avr}(f) \mid \mathcal{B}_{t}\left(W_{L}(M)\right)\right](\omega) \rightarrow \operatorname{Avr}(f)(\omega) \quad(t \rightarrow \infty)
\end{aligned}
$$

$P^{x}$-a.s. $\omega$ and $L^{1}\left(P^{x}\right)$ for $x \in M$. Therefore we have

$$
\begin{aligned}
\left\|A v r_{0}(f)-\operatorname{Avr}(f)\right\|_{L^{1}\left(P^{m}\right)} & =\left\|A v r_{0}(f) \circ \sigma_{t}-\operatorname{Avr}(f)\right\|_{L^{1}\left(P^{m}\right)} \\
& =\int_{M}\left\|A v r_{0}(f) \circ \sigma_{t}-A v r(f)\right\|_{L^{1}\left(P^{x}\right)} m(d x) \rightarrow 0 \quad(t \rightarrow \infty) .
\end{aligned}
$$

This implies that $P^{m}\left(Q_{2}\left(W_{L}(M)\right)\right)=1$.

(3) If $x \in Q_{X}$, then the map $c(x): f \mapsto \lim _{t \rightarrow \infty} \frac{1}{t} \int_{0}^{t} T(s) f(x) d s$ is a bounded positive linear functional on $C(M)$ with $c(x)(1)=1$. Therefore there exists a probability measure $m_{x}$ such that $c(x)(f)=\int_{M} f d m_{x}$ by the Riesz representation theorem. Substituting $T(t) f$ for $f$, we see that $m_{x}$ is an $A$-harmonic probability measure. 
For $x \in M$ and $f \in C(M)$ we define a stochastic process $Y_{\lambda}^{x}=\left\{Y_{\lambda}^{x}(t)\right\}_{t \geq 0}$ with parameter $\lambda$ by

$$
Y_{\lambda}^{x}(t)=\frac{1}{\sqrt{\lambda}} \int_{0}^{\lambda t} f\left(X^{x}(s)\right) d s .
$$

Now we can state a central limit theorem for the $A$-leafwise diffusion $X$.

THEOREM 2.8. For a real-valued function $h \in C_{L}^{2}(M)$ let $f=A$ and consider the process $Y_{\lambda}^{x}$ defined by (2.14). Then for any $x \in Q_{X}$, the processes $Y_{\lambda}^{x}$ converge in law to the Brownian motion $W_{\langle f\rangle(x)}$ with variance $\langle f\rangle(x)$ t for each time $t \geq 0$ as $\lambda \rightarrow \infty$, where $\langle f\rangle(x)$ is given by

$$
\langle f\rangle(x)=\int_{M}\left\|\operatorname{grad}_{L} h\right\|_{g}^{2} d m_{x},
$$

$g$ is the leafwise smooth Riemannian metric induced by $A$ and $m_{x}$ is the A-harmonic probability measure appearing in Proposition 2.7.

REMARK 2.9. (1) If there exists $y \in \operatorname{supp} m_{x}$ such that $\left\|\operatorname{grad}_{L} h(y)\right\|_{g}>0$, then $\langle f\rangle(x)>0$, i.e., the Brownian motion $W_{\langle f\rangle(x)}$ is non-degenerate. If $\langle f\rangle(x)=0$, then we regard the process $W_{\langle f\rangle(x)}$ as a process which is constantly 0 .

(2) For a stationary reversible Markov process, the same kind of central limit theorem as above under extremely general setting was proved by Kipnis and Varadhan in [9]. But as noted by themselves in [9, Remark 1.7], their idea is not directly applicable to the limit problem concerned with almost every starting point with respect to any harmonic measure.

3. Construction of leafwise diffusions on foliated spaces. The aim of this section is to prove Theorem 2.1. First we consider a stochastic differential equation on $\mathbb{R}^{d} \times Z$ given by

$$
d Y(t)=\sigma_{\alpha}\left(Y(t), Z_{0}\right) d B^{\alpha}(t)+\sigma_{0}\left(Y(t), Z_{0}\right) d t,
$$

where $\sigma_{0}, \sigma_{1}, \ldots, \sigma_{r}$ are $\mathbb{R}^{d}$-valued, bounded leafwise smooth maps on $\mathbb{R}^{d} \times Z$. Let $Y=$ $\{Y(t)\}_{t \geq 0}$ be an $\mathbb{R}^{d}$-valued, $\left(\mathcal{F}_{t}\right)$-adapted continuous process and $Z_{0}$ a $Z$-valued, $\mathcal{F}_{0}$-random variable on a usual filtered probability space $\left(\Omega, \mathcal{F}, P,\left(\mathcal{F}_{t}\right)_{t \geq 0}\right)$. We say that the pair $\left(Y, Z_{0}\right)$ is a solution of (3.1) if there exists an $r$-dimensional $\left(\mathcal{F}_{t}\right)$-Brownian motion $B=\{B(t)\}_{t \geq 0}$ such that

$$
Y(t)-Y(0)=\int_{0}^{t} \sigma_{\alpha}\left(Y(s), Z_{0}\right) d B^{\alpha}(s)+\int_{0}^{t} \sigma_{0}\left(Y(s), Z_{0}\right) d s \quad \text { for } t \geq 0 \quad P \text {-a.s. }
$$

The results summarized in the following lemma are rather elementary but they play important roles in the construction of the solutions of stochastic differential equations on foliated spaces. So we shall give their proofs for the sake of later convenience.

LEMMA 3.1. (i) The pathwise uniqueness of solutions for (3.1) holds, i.e., if $\left(Y_{1}, Z_{1}\right)$ and $\left(Y_{2}, Z_{2}\right)$ are solutions of (3.1) with an $r$-dimensional $\left(\mathcal{F}_{t}\right)$-Brownian motion on a usual filtered probability space $\left(\Omega, \mathcal{F}, P,\left(\mathcal{F}_{t}\right)_{t \geq 0}\right)$ and satisfy $\left(Y_{1}(0), Z_{1}\right)=\left(Y_{2}(0), Z_{2}\right) P$-a.s., then $Y_{1}=Y_{2} P$-a.s. 
(ii) For any $(y, z) \in \mathbb{R}^{d} \times Z$, there exists an $\mathbb{R}^{d}$-valued continuous stochastic process $Y^{(y, z)}=\left\{Y^{(y, z)}(t)\right\}_{t \geq 0}$ on the $r$-dimensional Winer space $\left(W_{0}^{r}, \mathcal{F}^{W}, P^{W},\left(\mathcal{F}_{t}^{W}\right)_{t \geq 0}\right)$ such that $\left(Y^{(y, z)}, z\right)$ is a solution of (3.1) with $\left(Y^{(y, z)}(0), z\right)=(y, z) P^{W}-a . s$.

(iii) For any $p \geq 1, T>0$ and compact subset $C$ of $\mathbb{R}^{d} \times Z$,

$$
\sup _{\substack{(y, z), \tilde{y}, \tilde{z}) \in C \\|y-\tilde{y}|+d_{Z}(z, \tilde{z})<\delta}} E\left[\sup _{0 \leq t \leq T}\left|Y^{(y, z)}(t)-Y^{(\tilde{y}, \tilde{z})}(t)\right|^{p}\right] \rightarrow 0 \quad(\delta \rightarrow 0),
$$

where $d_{Z}$ is a metric on $Z$.

PROOF. (i) Using the regular conditional probability given $\mathcal{F}_{0}$, we need only consider the case where $\left(Y_{1}(0), Z_{1}\right)=\left(Y_{2}(0), Z_{2}\right)=(y, z) P$-a.s. for some $(y, z) \in \mathbb{R}^{d} \times Z$ (see [6, Remark 1.4 in Chapter IV-1]). Then $Y_{1}$ and $Y_{2}$ are solutions of a stochastic differential equation on $\mathbb{R}^{d}$ given by

$$
\left\{\begin{array}{l}
d Y(t)=\sigma_{\alpha}(Y(t), z) d B^{\alpha}(t)+\sigma_{0}(Y(t), z) d t \\
Y(0)=y
\end{array} .\right.
$$

Since the maps $\sigma_{0}(\cdot, z), \sigma_{1}(\cdot, z), \ldots, \sigma_{r}(\cdot, z)$ are locally Lipschitz continuous, the pathwise uniqueness of solutions for (3.2) holds (see [6, Chapter IV-3] and [16, Chapter V-2] for examples). Therefore we have $Y_{1}=Y_{2} P$-a.s.

(ii) Obviously, the assertion (ii) is valid by the existence of a strong solution for the equation (3.2) (see [6, Chapter IV] and [8, Chapter 5-2 and 5-3]).

(iii) We assume that $d=r=1$ for simplicity. In the general case, we can also prove in the same way. Furthermore, we may assume that $p \geq 2$ and $C$ is of the form $[-K, K] \times Z^{\prime}$, where $K>0$ and $Z^{\prime}$ is a compact subset of $Z$. Take $T>0$ and fix it. In the following $K_{1}, K_{2}, \ldots$ are positive constants which may depend on $T, K$ and $p$. We first show that

$$
\sup _{(y, z) \in C} E\left[\sup _{0 \leq t \leq T}\left|Y^{(y, z)}(t)\right|^{p+1}\right]<\infty .
$$

We have

$$
\begin{aligned}
E\left[\sup _{0 \leq t \leq T}\left|Y^{(y, z)}(t)\right|^{p+1}\right] \leq K_{1}\{ & K^{p+1}+E\left[\sup _{0 \leq t \leq T}\left|\int_{0}^{t} \sigma_{1}\left(Y^{(y, z)}(s), z\right) d w(s)\right|^{p+1}\right] \\
& \left.+E\left[\sup _{0 \leq t \leq T}\left|\int_{0}^{t} \sigma_{0}\left(Y^{(y, z)}(s), z\right) d s\right|^{p+1}\right]\right\} .
\end{aligned}
$$

Here

$$
\begin{aligned}
E\left[\sup _{0 \leq t \leq T}\left|\int_{0}^{t} \sigma_{1}\left(Y^{(y, z)}(s), z\right) d w(s)\right|^{p}\right] & \leq K_{2} E\left[\left(\int_{0}^{t} \sigma_{1}\left(Y^{(y, z)}(s), z\right)^{2} d s\right)^{(p+1) / 2}\right] \\
& \leq K_{3} E\left[\int_{0}^{T}\left\|\sigma\left(Y^{(y, z)}(t), z\right)\right\|^{p+1} d t\right] \\
& \leq K_{3} T\left\|\sigma_{1}\right\|_{\infty}^{p+1}
\end{aligned}
$$


and

$$
E\left[\sup _{0 \leq t \leq T}\left|\int_{0}^{t} \sigma_{0}\left(Y^{(y, z)}(s), z\right) d s\right|^{p+1}\right] \leq K_{4} T\left\|\sigma_{0}\right\|_{\infty}^{p+1}
$$

from a well-known moment inequality [6, Theorem III-3.1] and the Hölder inequality. Thus we obtain

$$
\sup _{(y, z) \in C} E\left[\sup _{0 \leq t \leq T}\left|Y^{(y, z)}(t)\right|^{p+1}\right]<\infty .
$$

For a fixed $n \geq K$, we define a $\left(\mathcal{F}_{t}^{W}\right)$-stopping time by $\tau_{n}^{(y, z)}=\inf \left\{t \geq 0:\left|Y^{(y, z)}(t)\right| \geq n\right\}$ for $(y, z) \in \mathbb{R} \times Z$. Let $0<\varepsilon<1$ be given. Since $\sigma_{0}$ and $\sigma_{1}$ are leafwise smooth, there exist $0<\delta_{n, Z^{\prime}}<\varepsilon$ and $K_{n, Z^{\prime}}>1$ depending on $n$ and $Z^{\prime}$ such that

$$
\left|\sigma_{\alpha}\left(y_{1}, z_{1}\right)-\sigma_{\alpha}\left(y_{2}, z_{1}\right)\right|+\left|\sigma_{\alpha}\left(y_{2}, z_{1}\right)-\sigma_{\alpha}\left(y_{2}, z_{2}\right)\right|<K_{n, Z^{\prime}}\left|y_{1}-y_{2}\right|+\varepsilon
$$

for any $\left(y_{1}, z_{1}\right),\left(y_{2}, z_{2}\right) \in[-n, n] \times Z^{\prime}$ with $d_{Z}\left(z_{1}, z_{2}\right)<\delta_{n, Z^{\prime}}$ and $\alpha=0,1$. If $(y, z)$, $(\tilde{y}, \tilde{z}) \in C$ and $t$ satisfy $|y-\tilde{y}|+d_{Z}(z, \tilde{z})<\delta_{n, Z^{\prime}}$ and $t \leq T$, then

$$
\begin{aligned}
& \left|\sigma_{\alpha}\left(Y^{(y, z)}\left(t \wedge \tau_{n}^{(y, z)} \wedge \tau_{n}^{(\tilde{y}, \tilde{z})}\right), z\right)-\sigma_{\alpha}\left(Y^{(\tilde{y}, \tilde{z})}\left(t \wedge \tau_{n}^{(y, z)} \wedge \tau_{n}^{(\tilde{y}, \tilde{z})}\right), \tilde{z}\right)\right|^{p} \\
& \leq K_{5}\left\{\left|\sigma_{\alpha}\left(Y^{(y, z)}\left(t \wedge \tau_{n}^{(y, z)} \wedge \tau_{n}^{(\tilde{y}, \tilde{z})}\right), z\right)-\sigma_{\alpha}\left(Y^{(\tilde{y}, \tilde{z})}\left(t \wedge \tau_{n}^{(y, z)} \wedge \tau_{n}^{(\tilde{y}, \tilde{z})}\right), z\right)\right|^{p}\right. \\
& \left.\quad+\left|\sigma_{\alpha}\left(Y^{(\tilde{y}, \tilde{z})}\left(t \wedge \tau_{n}^{(y, z)} \wedge \tau_{n}^{(\tilde{y}, \tilde{z})}\right), z\right)-\sigma_{\alpha}\left(Y^{(\tilde{y}, \tilde{z})}\left(t \wedge \tau_{n}^{(y, z)} \wedge \tau_{n}^{(\tilde{y}, \tilde{z})}\right), \tilde{z}\right)\right|^{p}\right\} \\
& \leq K_{5} K_{n, Z^{\prime}}^{p}\left\{\left|Y^{(y, z)}\left(t \wedge \tau_{n}^{(y, z)} \wedge \tau_{n}^{(\tilde{y}, \tilde{z})}\right)-Y^{\tilde{y}, \tilde{z}}\left(t \wedge \tau_{n}^{(y, z)} \wedge \tau_{n}^{(\tilde{y}, \tilde{z})}\right)\right|^{p}+\varepsilon\right\}
\end{aligned}
$$

for $\alpha=0,1$. Therefore we have

$$
\begin{aligned}
& E\left[\sup _{0 \leq s \leq t \wedge \tau_{n}^{(y, z)} \wedge \tau_{n}^{(\tilde{y}, \tilde{z})}}\left|Y^{(y, z)}(s)-Y^{(\tilde{y}, \tilde{z})}(s)\right|^{p}\right] \\
& \leq K_{6}\left\{|y-\tilde{y}|^{p}+E\left[\sup _{0 \leq s \leq t \wedge \tau_{n}^{(y, z)} \wedge \tau_{n}^{(\tilde{y}, \tilde{z})}}\left|\int_{0}^{s}\left\{\sigma_{1}\left(Y^{(y, z)}(u), z\right)-\sigma_{1}\left(Y^{(\tilde{y}, \tilde{z})}(u), \tilde{z}\right)\right\} d w(u)\right|^{p}\right]\right. \\
& \left.+E\left[\sup _{0 \leq s \leq t \wedge \tau_{n}^{(y, z)} \wedge \tau_{n}^{(\tilde{y}, \tilde{z})}}\left|\int_{0}^{s}\left\{\sigma_{0}\left(Y^{(y, z)}(u), z\right)-\sigma_{0}\left(Y^{(\tilde{y}, \tilde{z})}(u), \tilde{z}\right)\right\} d u\right|^{p}\right]\right\} \\
& \leq K_{6}|y-\tilde{y}|^{p} \\
& +K_{7} E\left[\int_{0}^{t} \mid \sigma_{1}\left(Y^{(y, z)}\left(s \wedge \tau_{n}^{(y, z)} \wedge \tau_{n}^{(\tilde{y}, \tilde{z})}\right), z\right)-\sigma_{1}\left(\left.Y^{(\tilde{y}, \tilde{z})}\left(s \wedge \tau_{n}^{(y, z)} \wedge \tau_{n}^{(\tilde{y}, \tilde{z})}, \tilde{z}\right)\right|^{p} d s\right]\right. \\
& +K_{8} E\left[\int_{0}^{t}\left|\sigma_{0}\left(Y^{(y, z)}\left(s \wedge \tau_{n}^{(y, z)} \wedge \tau_{n}^{(\tilde{y}, \tilde{z})}\right), z\right)-\sigma_{0}\left(Y^{(\tilde{y}, \tilde{z})}\left(s \wedge \tau_{n}^{(y, z)} \wedge \tau_{n}^{(\tilde{y}, \tilde{z})}\right), \tilde{z}\right)\right|^{p} d s\right] \\
& <K_{9} K_{n, Z^{\prime}}^{p}\left\{\varepsilon+\int_{0}^{t} E\left[\left|Y^{(y, z)}\left(s \wedge \tau_{n}^{(y, z)} \wedge \tau_{n}^{(\tilde{y}, \tilde{z})}\right)-Y^{(\tilde{y}, \tilde{z})}\left(s \wedge \tau_{n}^{(y, z)} \wedge \tau_{n}^{(\tilde{y}, \tilde{z})}\right)\right|^{p}\right] d s\right\} .
\end{aligned}
$$

By the Gronwall inequality, we obtain

$$
E\left[\sup _{0 \leq t \leq T \wedge \tau_{n}^{(y, z)} \wedge \tau_{n}^{(\tilde{y}, \tilde{z})}}\left|Y^{(y, z)}(t)-Y^{(\tilde{y}, \tilde{z})}(t)\right|^{p}\right] \leq \varepsilon K_{10} K_{n, Z^{\prime}}^{p} \exp \left\{K_{10} K_{n, Z^{\prime}}^{p} T\right\} .
$$


Consequently, for any $n \geq K$,

$$
\sup _{\substack{(y, z),(\tilde{y}, \tilde{z}) \in C \\|y-\tilde{y}|+d z(z, \tilde{z})<\delta}} E\left[\sup _{0 \leq t \leq T \wedge \tau_{n}^{(y, z)} \wedge \tau_{n}^{(\tilde{y}, \tilde{z})}}\left|Y^{(y, z)}(t)-Y^{(\tilde{y}, \tilde{z})}(t)\right|^{p}\right] \rightarrow 0 \quad(\delta \rightarrow 0) .
$$

In addition, we have

$$
\begin{aligned}
& E\left[\sup _{0 \leq t \leq T}\left|Y^{(y, z)}(t)-Y^{(\tilde{y}, \tilde{z})}(t)\right|^{p}\right] \\
& \leq E\left[\sup _{\left.0 \leq t \leq T \wedge \tau_{n}^{(y, z)} \wedge \tau_{n}^{(\tilde{y}, \tilde{z})}\left|Y^{(y, z)}(t)-Y^{(\tilde{y}, \tilde{z})}(t)\right|^{p}\right]}\right. \\
&+E\left[\sup _{0 \leq t \leq T}\left(\left|Y^{(y, z)}(t)\right|+\left|Y^{(\tilde{y}, \tilde{z})}(t)\right|\right)^{p}: \tau_{n}^{(y, z)} \wedge \tau_{n}^{(\tilde{y}, \tilde{z})} \leq T\right] \\
& \leq E\left[\sup _{\left.0 \leq t \leq T \wedge \tau_{n}^{(y, z)} \wedge \tau_{n}^{(\tilde{y}, \tilde{z})}\left|Y^{(y, z)}(t)-Y^{(\tilde{y}, \tilde{z})}(t)\right|^{p}\right]}\right. \\
&+E\left[\sup _{0 \leq t \leq T}\left(\left|Y^{(y, z)}(t)\right|+\left|Y^{(\tilde{y}, \tilde{z})}(t)\right|\right)^{p}: \sup _{0 \leq t \leq T}\left(\left|Y^{(y, z)}(t)\right|+\left|Y^{(\tilde{y}, \tilde{z})}(t)\right|\right) \geq n\right] \\
& \leq E\left[\sup _{\left.0 \leq t \leq T \wedge \tau_{n}^{(y, z)} \wedge \tau_{n}^{(\tilde{y}, \tilde{z})}\left|Y^{(y, z)}(t)-Y^{(\tilde{y}, \tilde{z})}(t)\right|^{p}\right]}\right] \\
&+\frac{1}{n} E\left[\sup _{0 \leq t \leq T}\left(\left|Y^{(y, z)}(t)\right|+\left|Y^{(\tilde{y}, \tilde{z})}(t)\right|\right)^{p+1}\right]
\end{aligned}
$$

for $(y, z),(\tilde{y}, \tilde{z}) \in C$. Combining (3.3) and (3.4), we obtain the desired result.

Let $W^{d}$ be the totality of $\mathbb{R}^{d}$-valued continuous maps on $[0, \infty)$ and $P^{(y, z)}$ the law of $W^{d} \times Z$-valued random variable $\left(Y^{(y, z)}, z\right)$. If $\mu$ is a Borel probability measure on $\mathbb{R}^{d} \times Z$, then a probability measure $P^{\mu}$ on $W^{d} \times Z$ is defined by $P^{\mu}(d \omega)=P^{(y, z)}(d \omega) \mu(d(y, z))$. We can easily show that the measure $P^{\mu}$ is the law of a solution of the equation (3.1) with the initial distribution $\mu$. Therefore by combining this result with Lemma 3.1-(i), we have a unique strong solution of the equation (3.1).

LEMMA 3.2. There exists a map $\bar{F}: \mathbb{R}^{d} \times Z \times W_{0}^{r} \rightarrow W^{d}$ satisfying the following:

(1) $\bar{F}$ is $\widehat{\mathcal{F}}\left(\mathbb{R}^{d} \times Z\right) / \mathcal{B}\left(W^{d}\right)$-measurable.

(2) $\bar{F}$ is $\widehat{\mathcal{F}}_{t}\left(\mathbb{R}^{d} \times Z\right) / \mathcal{B}_{t}\left(W^{d}\right)$-measurable.

(3) Suppose that $\left(Y, Z_{0}\right)$ is a solution of (3.1) with an $\left(\mathcal{F}_{t}\right)$-Brownian motion $B=$ $\left\{B_{t}\right\}_{t \geq 0}$ on a usual filtered probability space $\left(\Omega, \mathcal{F}, P,\left(\mathcal{F}_{t}\right)_{t \geq 0}\right)$.

Then $Y=\bar{F}\left(Y(0), Z_{0}, B\right) P$-a.s.

(4) If $B=\{B(t)\}$ is an $\left(\mathcal{F}_{t}\right)$-Brownian motion, $\xi$ is an $\mathbb{R}^{d}$-valued $\mathcal{F}_{0}$-random variable and $\eta$ is a $Z$-valued $\mathcal{F}_{0}$-random variable on $\left(\Omega, \mathcal{F}, P,\left(\mathcal{F}_{t}\right)_{t \geq 0}\right)$, then $(\bar{F}(\xi, \eta, B), \eta)$ is a solution of (3.1) on $\left(\Omega, \mathcal{F}, P,\left(\mathcal{F}_{t}\right)_{t \geq 0}\right)$ with the initial value $(\xi, \eta)$. 
We omit the proof since it is shown in the same way as [6, Theorem IV-1.1] and [8, Corollary 5-3.23].

Recall that we consider the stochastic differential equation on $M$ given by

$$
d X(t)=A_{\alpha}(X(t)) \circ d B^{\alpha}(t)+A_{0}(X(t)) d t,
$$

where $A_{0}, A_{1}, \ldots, A_{r}$ are leafwise smooth vector fields. First we construct "local" solutions of (3.5). For a fixed foliated chart $(U,(y, z))$, these vector fields are expressed as

$$
A_{\alpha}=\sigma_{\alpha}^{i}(y, z) \frac{\partial}{\partial y^{i}}, \quad \alpha=0,1, \ldots, r
$$

in $(U,(y, z))$. We can extend $\sigma_{0}, \sigma_{1}, \ldots, \sigma_{r}$ to leafwise smooth functions with compact support on $\mathbb{R}^{d} \times Z$. Consider a stochastic differential equation on $\mathbb{R}^{d} \times Z$ given by

$$
\begin{aligned}
d Y(t) & =\sigma_{\alpha}(Y(t), z) \circ d B^{\alpha}(t)+\sigma_{0}(Y(t), z) d t \\
& =\sigma_{\alpha}(Y(t), z) d B^{\alpha}(t)+\bar{\sigma}_{0}(Y(t), z) d t,
\end{aligned}
$$

where

$$
\bar{\sigma}_{0}^{i}(y, z)=\sigma_{0}^{i}(y, z)+\frac{1}{2} \sum_{\alpha=1}^{r}\left(\frac{\partial \sigma_{\alpha}^{i}}{\partial y^{k}}(y, z)\right) \sigma_{\alpha}^{k}(y, z) .
$$

Then we have the strong solution $\bar{F}_{U}: \mathbb{R}^{d} \times Z \times W_{0}^{r} \rightarrow W^{d}$ for the equation (3.6) by Lemma 3.2. We notice that the map $(y, z): U \rightarrow B_{1} \times B_{2}$ is extended to a map from $\bar{U}$ onto $\overline{B_{1}} \times \overline{B_{2}}$. Define maps $\tau_{B_{1} \times B_{2}}^{(y, z)}, F_{U}$ and $\tau_{U}$ so that

$$
\begin{aligned}
\tau_{B_{1} \times B_{2}}^{(y, z)}(w) & =\inf \left\{t \geq 0:\left(\bar{F}_{U}(y, z, w)(t), z\right) \notin B_{1} \times B_{2}\right\}, \\
F_{U}(t, x, w) & =(y, z)^{-1}\left(\bar{F}_{U}(y(x), z(x), w)\left(t \wedge \tau_{B_{1} \times B_{2}}^{(y(x), z(x))}\right), z(x)\right) \text { and } \\
\tau_{U}(x, w) & =\tau_{B_{1} \times B_{2}}^{(\varphi(x))}(w) .
\end{aligned}
$$

Recall that the rule (2.2) for $A_{0}, A_{1}, \ldots, A_{r}$ under changes of coordinates and the fact that the chain rule for the operation $\circ$ takes the same form as in the ordinary calculus. Then we can show that if $V \in \mathcal{U}$ is another foliated chart containing $x, F_{U}(t, x)=F_{V}(t, x)$ for $t \leq \tau_{U}(x) \wedge \tau_{V}(x) P^{W}$-a.s. by the pathwise uniqueness of solutions for the equation (3.6).

Next we patch together the local solutions. Let $\mathcal{U}=\left\{\left(U_{\alpha}, \varphi_{\alpha}\right)\right\}$ be a foliated atlas of $M$. We may assume that for any $\alpha$, there exist foliated charts $\left(U_{\alpha, 1}, \varphi_{\alpha, 1}\right)$ and $\left(U_{\alpha, 2}, \varphi_{\alpha, 2}\right)$ such that $\overline{U_{\alpha}} \subset U_{\alpha, 1} \subset \overline{U_{\alpha, 1}} \subset U_{\alpha, 2},\left.\varphi_{\alpha, 2}\right|_{U_{\alpha, 1}}=\varphi_{\alpha, 1}$ and $\left.\varphi_{\alpha, 1}\right|_{U_{\alpha}}=\varphi_{\alpha}$. For $x \in M$, if $\left\{U_{1}, U_{2}, \ldots, U_{l}\right\}$ is the totality of foliated charts in $\mathcal{U}$ containing $x$, we set $\widehat{\tau}(x, w)=$ $\max \left\{\tau_{U_{i, 1}}(x, w): 1 \leq i \leq l\right\}$. Then a map $\widehat{F}$ is defined for $t \leq \widehat{\tau}(x, w)$ so that $\widehat{F}(t, x, w)=$ $F_{U_{i, 1}}(t, x, w)$ for $t \leq \tau_{U_{i, 1}}(x, w)$ and $1 \leq i \leq l P^{W}$-a.s.w. Define $\tau_{1}=\widehat{\tau}$ and $F(t)=$ $\widehat{F}(t)$ for $t \leq \tau_{1}$. Inductively, if $\tau_{n}$ and $F(t)$ are defined for $0 \leq t \leq \tau_{n}$, then on the set $\left\{(x, w): \tau_{n}(x, w)<\infty\right\}$, we define

$$
x_{n}=F\left(\tau_{n}\right), \quad w_{n}=\theta_{\tau_{n}} w, \quad \tau_{n+1}=\tau_{n}+\widehat{\tau}\left(x_{n}, w_{n}\right) \quad \text { and } \quad F(t)=\widehat{F}\left(t-\tau_{n}, x_{n}, w_{n}\right)
$$

for $\tau_{n} \leq t \leq \tau_{n+1}$. Thus $F(t)$ is defined for $t<\lim _{n \rightarrow \infty} \tau_{n}$.

Now we prove Theorem 2.1. 
Proof of Theorem 2.1. We divide the proof into three steps.

(Step 1) For any $x \in M, P^{W}\left(\tau_{n}(x) \uparrow \infty(n \uparrow \infty)\right)=1$.

Take $x \in M$ and fix it. By definition, $\widehat{\tau}(x), \tau_{n}(x), n=1,2, \ldots$ are $\left(\mathcal{F}_{t}^{W}\right)$-stopping times. We need to show that

$$
E\left[\prod_{n} 1_{\left\{\tau_{n}(x)<\infty\right\}} \exp \left(-\widehat{\tau}\left(x_{n}, w_{n}\right)\right)\right]=0 .
$$

Since $M$ is compact, we may assume that $\mathcal{U}$ is finite. We can prove that there exists $k<1$ such that

$$
1_{\left\{\tau_{n}(x)<\infty\right\}} E\left[\exp \left(-\widehat{\tau}\left(x_{n}, w_{n}\right)\right) \mid \mathcal{F}_{\tau_{n}(x)}^{W}\right] \leq k
$$

for any $n \geq 1$ and $x \in M$ in the same way as [6, Lemma IV-2.1]. Moreover, we have

$$
\begin{aligned}
E\left[\prod_{n=1}^{m+1} 1_{\left\{\tau_{n}(x)<\infty\right\}} \exp \left(-\widehat{\tau}\left(x_{n}, w_{n}\right)\right)\right] & =E\left[E\left[\prod_{n=1}^{m+1} 1_{\left\{\tau_{n}(x)<\infty\right\}} \exp \left(-\widehat{\tau}\left(x_{n}, w_{n}\right)\right) \mid \mathcal{F}_{\tau_{m+1}(x)}^{W}\right]\right] \\
& \leq k E\left[\prod_{n=1}^{m} 1_{\left\{\tau_{n}(x)<\infty\right\}} \exp \left(-\widehat{\tau}\left(x_{n}, w_{n}\right)\right)\right]
\end{aligned}
$$

for $m \geq 1$. Therefore we reach the desired result.

(Step 2) The map $F:(x, w) \mapsto F(\cdot, x, w)$ gives a unique strong solution for (2.5) ((3.5)), i.e., $F$ satisfies (1)-(4) in Theorem 2.1 .

The assertions (1) and (2) follow from the measurability of the maps $\left\{\bar{F}_{U_{\alpha, 1}}\right\}$ stated in Lemma 3.2. To verify the assertion (4) we need only to show in the case where $(\Omega, \mathcal{F}, P$, $\left.\left(\mathcal{F}_{t}\right)_{t \geq 0}\right)=\left(M \times W_{0}^{r}, \mathcal{F}(M)^{\mu}, \mu \otimes P^{W},\left(\mathcal{F}_{t}(M)^{\mu}\right)_{t \geq 0}\right), B(t)(x, w)=w(t)$ and $\xi(x, w)=$ $x$ for a fixed Borel probability measure $\mu$ on $M$. Suppose that $x \in U_{\alpha}$ and $f \in C_{L}^{2}(M)$. Since $\bar{F}_{U_{\alpha, 1}}$ is a solution of the equation (3.6), we have

$$
\begin{aligned}
f(F(t & \left.\left.\wedge \tau_{1}(x)\right)\right)-f(x) \\
= & \int_{0}^{t \wedge \tau_{1}(x)}\left(\sigma_{\alpha}^{i} \frac{\partial f}{\partial y^{i}}\right)\left(\bar{F}_{U_{\alpha, 1}}(y(x), z(x))(s), z(x)\right) \circ d w^{\alpha}(s) \\
& +\int_{0}^{t \wedge \tau_{1}(x)}\left(\sigma_{0}^{i} \frac{\partial f}{\partial y^{i}}\right)\left(\bar{F}_{U_{\alpha, 1}}(y(x), z(x))(s), z(x)\right) d s \\
= & \int_{0}^{t \wedge \tau_{1}(x)}\left(A_{\alpha} f\right)(F(s, x)) \circ d w^{\alpha}(s)+\int_{0}^{t \wedge \tau_{1}(x)}\left(A_{0} f\right)(F(s, x)) d s P^{W} \text {-a.s. }
\end{aligned}
$$

on the set $\left\{w: \tau_{1}(x, w)=\tau_{U_{\alpha, 1}}(x, w)\right\}$. Therefore

$$
f\left(F\left(t \wedge \tau_{1}\right)\right)-f(F(0))=\int_{0}^{t \wedge \tau_{1}}\left(A_{\alpha} f\right)(F(s)) \circ d w^{\alpha}(s)+\int_{0}^{t \wedge \tau_{1}}\left(A_{0} f\right)(F(s)) d s
$$

$\mu \otimes P^{W}$-a.s. Substituting the law of $x_{n}$ for $\mu$ and noticing that $x_{n}$ and $w_{n}$ are independent for $n \geq 1$, we see that

$$
f\left(F\left(t \wedge \tau_{n+1}\right)\right)-f\left(F\left(t \wedge \tau_{n}\right)\right)=\int_{t \wedge \tau_{n}}^{t \wedge \tau_{n+1}}\left(A_{\alpha} f\right)(F(s)) \circ d w^{\alpha}(s)
$$




$$
+\int_{t \wedge \tau_{n}}^{t \wedge \tau_{n+1}}\left(A_{0} f\right)(F(s)) d s
$$

$\mu \times P^{W}$-a.s. From Step 1, it follows that $\tau_{n} \uparrow \infty(n \uparrow \infty) \mu \otimes P^{W}$-a.s. Hence we see that the assertion (4) is valid. It remains to prove the assertion (3). But it immediately follows by the pathwise uniqueness of solutions for the equations (3.6).

We define a map $X^{x}: W_{0}^{r} \rightarrow W_{L}(M)$ for a fixed $x \in M$ by $X^{x}(t, w)=F(x, w)(t)$ for $(t, w) \in[0, \infty) \times W_{0}^{r}$. Let $d_{M}$ be a metric on $M$.

(Step 3) For any $\varepsilon>0$ and $T>0$, there exists $\delta>0$ such that

$$
P^{W}\left(\sup _{0 \leq t \leq T} d_{M}\left(X^{x}(t), X^{\tilde{x}}(t)\right)<\varepsilon\right) \geq 1-\varepsilon
$$

for any $x, \tilde{x} \in M$ with $d_{M}(x, \tilde{x})<\delta$.

We take a foliated atlas $\mathcal{U}=\left\{\left(U_{i}, \varphi_{i}\right)\right\}_{i=1}^{m}$ of $M$ satisfying that there exist foliated charts $\left(U_{i, 1}, \varphi_{i, 1}\right)$ and $\left(U_{i, 2}, \varphi_{i, 2}\right)$ such that $\overline{U_{i}} \subset U_{i, 1} \subset \overline{U_{i, 1}} \subset U_{i, 2},\left.\varphi_{i, 2}\right|_{U_{i, 1}}=\varphi_{i, 1}$ and $\left.\varphi_{i, 1}\right|_{U_{i}}=\varphi_{i}$ for each $i$. Let $l$ be a Lebesgue number of $\mathcal{U}$. For each $x \in M$ we put $\mathcal{I}(x)=\left\{1 \leq i \leq m: B_{x}(l / 2) \subset U_{i}\right\}$ and $i(x)=\min \mathcal{I}(x)$, where $B_{x}(l / 2)$ is the open ball of radius $l / 2$ centered at $x$ in $M$. We define maps by

$\rho(x, w)=\inf \left\{t \geq 0: X(t, x, w) \notin U_{i(x), 1}\right\}, \sigma(x, w)=\inf \left\{t \geq 0: X(t, x, w) \notin U_{i(x), 2}\right\}$ for $(x, w) \in M \times W_{0}^{r}$,

$$
\begin{aligned}
& \rho(\tilde{x} ; x, w)=\inf \left\{t \geq 0: X(t, \tilde{x}, w) \notin U_{i(x), 1}\right\}, \\
& \sigma(\tilde{x} ; x, w)=\inf \left\{t \geq 0: X(t, \tilde{x}, w) \notin U_{i(x), 2}\right\}
\end{aligned}
$$

for $(x, \tilde{x}, w) \in M \times M \times W_{0}^{r}$ and $\rho_{0}(x, w)=0, \rho_{n+1}(x, w)=\rho_{n}(x, w)+\rho\left(X^{x}\left(\rho_{n}\right), \theta_{\rho_{n}} w\right)$ for $n \geq 0$.

To prove the assertion of Step 3, we need the next lemma.

Lemma 3.3. (i) For any $x \in M, P^{W}\left(\rho_{n}(x) \uparrow \infty(n \uparrow \infty)\right)=1$.

(ii) For any $\varepsilon>0$ and $T>0$, there exists $\delta>0$ such that

$$
P^{W}\left(\sup _{0 \leq t \leq T} d_{M}\left(X^{x}(t \wedge \rho(x)), X^{\tilde{x}}(t \wedge \rho(x))\right)<\varepsilon\right) \geq 1-\varepsilon
$$

for any $x, \tilde{x} \in M$ with $d_{M}(x, \tilde{x})<\delta$.

Proof of Lemma 3.3. The assertion (i) immediately follows from the same reason as Step 1 stated above. The assertion (ii) is shown as follows. Put

$$
D=\min \left\{d_{M}\left(\overline{U_{i, 1}}, M \backslash U_{i, 2}\right): 1 \leq i \leq m\right\} .
$$

We take any $T>0$ and $0<\varepsilon<D / 2$. From Lemma 3.1-(iii) and the Chebyshev inequality in each $U_{i, 2}$, we see that there exists $0<\delta<l / 2$ such that

$$
P^{W}\left(\sup _{0 \leq t \leq T} d_{M}\left(X^{x}(t \wedge \sigma(x) \wedge \sigma(\tilde{x} ; x)), X^{\tilde{x}}(t \wedge \sigma(x) \wedge \sigma(\tilde{x} ; x))\right)<\varepsilon\right) \geq 1-\varepsilon
$$


whenever $d_{M}(x, \tilde{x})<\delta$. Since $\varepsilon<D / 2$, if $d_{M}(x, \tilde{x})<\delta$ and

$$
\sup _{0 \leq t \leq T} d_{M}\left(X^{x}(t \wedge \sigma(x) \wedge \sigma(\tilde{x} ; x), w), X^{\tilde{x}}(t \wedge \sigma(x) \wedge \sigma(\tilde{x} ; x), w)\right)<\varepsilon,
$$

then $\rho(x, w) \leq(\sigma(x) \wedge \sigma(\tilde{x} ; x))(w)$. Therefore the inequality (3.7) implies

$$
P^{W}\left(\sup _{0 \leq t \leq T} d_{M}\left(X^{x}(t \wedge \rho(x)), X^{\tilde{x}}(t \wedge \rho(x))\right)<\varepsilon\right) \geq 1-\varepsilon,
$$

which completes the proof of Lemma 3.3.

Now we return to the proof of Theorem 2.1. Let $\varepsilon>0, T>0$ and $x \in M$ be given. It suffices to show that there exists $\delta>0$ such that $P^{W}\left(\sup _{0 \leq t \leq T} d_{M}\left(X^{x}(t), X^{\tilde{x}}(t)\right)<\varepsilon\right) \geq$ $1-\varepsilon$ for any $\tilde{x} \in M$ with $d_{M}(x, \tilde{x})<\delta$. By Lemma 3.3-(ii), we can choose $0<\delta_{1}<\varepsilon$ so that

$$
P^{W}\left(\sup _{0 \leq t \leq T \wedge \rho_{1}\left(x_{1}\right)} d_{M}\left(X^{x_{1}}(t), X^{x_{2}}(t)\right)<\varepsilon\right) \geq 1-\varepsilon
$$

for any $x_{1}, x_{2} \in M$ with $d_{M}\left(x_{1}, x_{2}\right)<\delta_{1}$. Applying Lemma 3.3-(ii) again, we can take $0<\delta_{2}<\delta_{1}$ so that

$$
P^{W}\left(\sup _{0 \leq t \leq T \wedge \rho_{1}\left(x_{1}\right)} d_{M}\left(X\left(t, x_{1}\right), X\left(t, x_{2}\right)\right)<\delta_{1}\right) \geq 1-\delta_{1}
$$

whenever $d_{M}\left(x_{1}, x_{2}\right)<\delta_{2}$. Since $\left(X^{x}\left(\rho_{1}(x)\right), X^{\tilde{x}}\left(\rho_{1}(x)\right)\right)$ and $\theta_{\rho_{1}(x)} w$ are independent on $\left(\rho_{1}(x)<\infty\right)$, we have

$$
\begin{aligned}
& P^{W}\left(\sup _{0 \leq t \leq T \wedge \rho_{2}(x)} d_{M}\left(X^{x}(t), X^{\tilde{x}}(t)\right)<\varepsilon\right) \\
&= P^{W}\left(\left(\sup _{0 \leq t \leq T \wedge \rho_{2}(x)} d_{M}\left(X^{x}(t), X^{\tilde{x}}(t)\right)<\varepsilon\right) \cap\left(T<\rho_{1}(x)\right)\right) \\
&+P^{W}\left(\left(\sup _{0 \leq t \leq T \wedge \rho_{2}(x)} d_{M}\left(X^{x}(t), X^{\tilde{x}}(t)\right)<\varepsilon\right) \cap\left(\rho_{1}(x) \leq T\right)\right) \\
& \geq P^{W}\left(\left(\sup _{0 \leq t \leq T \wedge \rho_{1}(x)} d_{M}\left(X^{x}(t), X^{\tilde{x}}(t)\right)<\varepsilon\right) \cap\left(T<\rho_{1}(x)\right)\right) \\
&+P^{W}\left(\left(\sup _{0 \leq t \leq \rho_{1}(x)} d_{M}\left(X^{x}(t), X^{\tilde{x}}(t)\right)<\delta_{1}\right) \cap\left(\rho_{1}(x) \leq T\right)\right. \\
&\left.\bigcap\left(\sup _{0 \leq t \leq T \wedge \rho_{1}\left(X^{x}\left(\rho_{1}(x)\right), \theta_{\rho_{1}(x)} w\right)} d_{M}\left(X\left(t, X^{x}\left(\rho_{1}(x)\right), \theta_{\rho_{1}(x)} w\right), X\left(t, X^{\tilde{x}}\left(\rho_{1}(x)\right), \rho_{\rho_{1}(x)} w\right)\right)<\varepsilon\right)\right) \\
& \geq P^{W}\left(\left(T<\rho_{1}(x)\right)\right)-\varepsilon \\
&+(1-\varepsilon) P^{W}\left(\left(\sup _{0 \leq t \leq \rho_{1}(x)} d_{M}\left(X^{x}(t), X^{\tilde{x}}(t)\right)<\delta_{1}\right) \cap\left(\rho_{1}(x) \leq T\right)\right) \\
& \geq P^{W}\left(\left(T<\rho_{1}(x)\right)\right)-\varepsilon+(1-\varepsilon)\left(P^{W}\left(\left(\rho_{1}(x) \leq T\right)\right)-\delta_{1}\right) \geq 1-3 \varepsilon
\end{aligned}
$$


if $d_{M}(x, \tilde{x})<\delta_{2}$. Here the first inequality follows from the fact that

$$
X^{\tilde{x}}\left(t+\rho_{1}(x)\right)=X\left(t, X^{\tilde{x}}\left(\rho_{1}(x)\right), \theta_{\rho_{1}(x)} w\right) \quad \text { for } t \geq 0 \quad P^{W} \text {-a.s. on }\left(\rho_{1}(x)<\infty\right) .
$$

Using the same argument above, we can show that for any positive integer $n$, there exists $\delta_{n}>0$ such that

$$
P^{W}\left(\sup _{0 \leq t \leq T \wedge \rho_{n}(x)} d_{M}\left(X^{x}(t), X^{\tilde{x}}(t)\right)<\varepsilon\right) \geq 1-\frac{\varepsilon}{2}
$$

for any $\tilde{x} \in M$ with $d_{M}(x, \tilde{x})<\delta_{n}$. From (i) of Lemma 3.3, there exists $N \geq 1$ such that $P^{W}\left(T \leq \rho_{N}(x)\right) \geq 1-\varepsilon / 2$. Consequently, we obtain

$$
\begin{aligned}
& P^{W}\left(\sup _{0 \leq t \leq T} d_{M}\left(X^{x}(t), X^{\tilde{x}}(t)\right)<\varepsilon\right) \\
& \quad \geq P^{W}\left(\left(\sup _{0 \leq t \leq T \wedge \rho_{N}(x)} d_{M}\left(X^{x}(t), X^{\tilde{x}}(t)\right)<\varepsilon\right) \cap\left(T \leq \rho_{N}(x)\right)\right) \\
& \quad \geq 1-\frac{\varepsilon}{2}-\frac{\varepsilon}{2}=1-\varepsilon
\end{aligned}
$$

whenever $d_{M}(x, \tilde{x})<\delta_{N}$. The proof of Theorem 2.1 is now complete.

REMARK 3.4. (1) Considering functions defined by $f(y, z)=y^{i}, i=1,2, \ldots, d$ in each foliated chart, we easily see that if a stochastic process $X=\{X(t)\}_{t \geq 0}$ satisfies the equality (2.6) for any $f \in C_{L}^{\infty}(M)$, then $X$ is a solution of the equation (2.5).

(2) Let $X_{L}^{x}=\left\{X_{L}^{x}(t)\right\}_{t \geq 0}$ be a solution on $\left(W_{0}^{r}, \mathcal{F}^{W}, P^{W},\left(\mathcal{F}_{t}^{W}\right)_{t \geq 0}\right)$ of the equation

$$
d X_{L}(t)=\left.A_{\alpha}\right|_{L}\left(X_{L}(t)\right) \circ d B^{\alpha}(t)+\left.A_{0}\right|_{L}\left(X_{L}(t)\right) d t
$$

on a leaf $L$ with the initial distribution $\delta_{x}$ and $i_{L}: L \rightarrow M$ the inclusion map. Consequently, we have $X^{x}=i_{L}\left(X_{L}^{x}\right) P^{W}$-a.s. by the pathwise uniquness of solutions in each foliated chart.

(3) If $M$ is non-compact, we can similarly construct a unique strong solution of (2.5) up to explosion times.

(4) As we mentioned in Remark 2.2, the stochastic continuity of the family $\left\{X^{x}\right\}_{x \in M}$ with respect to $x$ is an important result to show its Feller property.

4. Central limit theorem for additive functionals. In this section we prove Theorem 2.8. Let $X=\left\{X^{x}\right\}_{x \in M}$ be an $A$-leafwise diffusion defined by (2.12). Let $x$ be an element in $M$ and $f$ a continuous function given by $f=A h$ for a function $h \in C_{L}^{2}(M)$. We consider the stochastic process $Y_{\lambda}^{x}=\left\{Y_{\lambda}^{x}(t)\right\}_{t \geq 0}$ defined by (2.14). If the process $\{r(t, r)\}_{t \geq 0}$ is a solution of the equation (2.11) on $O(\mathcal{L})$ satisfying that $\pi(r(t, r))=X^{x}(t)$ for $t \geq 0$, then we have

$$
\begin{aligned}
& h\left(X^{x}(t)\right)-h(x)=h \circ \pi(r(t, r))-h \circ \pi(r) \\
& \quad=\int_{0}^{t} \widetilde{H}_{\alpha}(h \circ \pi)(r(s, r)) \circ d w^{\alpha}(s)+\int_{0}^{t} \widetilde{H}_{0}(h \circ \pi)(r(s, r)) d s
\end{aligned}
$$




$$
\begin{aligned}
& =\int_{0}^{t} \widetilde{H}_{\alpha}(h \circ \pi)(r(s, r)) d w^{\alpha}(s)+\int_{0}^{t}\left(\frac{1}{2} \sum_{\alpha=1}^{d} \widetilde{H}_{\alpha} \widetilde{H}_{\alpha}+\widetilde{H}_{0}\right)(h \circ \pi)(r(s, r)) d s \\
& =\int_{0}^{t} \widetilde{H}_{\alpha}(h \circ \pi)(r(s, r)) d w^{\alpha}(s)+\int_{0}^{t} f\left(X^{x}(s)\right) d s .
\end{aligned}
$$

We put

$$
M_{\lambda}^{x}(t)=\frac{-1}{\sqrt{\lambda}} \int_{0}^{\lambda t} \widetilde{H}_{\alpha}(h \circ \pi)(r(s, r)) d w^{\alpha}(s)
$$

for $t \geq 0$. Then we have

$$
Y_{\lambda}^{x}(t)=M_{\lambda}^{x}(t)+\frac{h\left(X^{x}(\lambda t)\right)-h(x)}{\sqrt{\lambda}} .
$$

In addition we see that the stochastic processes $\left\{M_{\lambda}^{x}\right\}$ are continuous martingales and their quadratic variations are given by

$$
\left\langle M_{\lambda}^{x}\right\rangle(t)=\frac{1}{\lambda} \int_{0}^{\lambda t} \sum_{\alpha=1}^{d}\left(\widetilde{H}_{\alpha}(h \circ \pi)(r(s, r))\right)^{2} d s=\frac{1}{\lambda} \int_{0}^{\lambda t}\left\|\operatorname{grad}_{L} h\left(X^{x}(s)\right)\right\|_{g}^{2} d s,
$$

where $g$ is the leafwise smooth Riemannian metric on $M$ induced by $A$. Note that

$$
\begin{aligned}
\sum_{\alpha=1}^{d}\left(\widetilde{H}_{\alpha}(h \circ \pi)\left(y, z,\left(e_{j}^{i}\right)\right)\right)^{2}=\sum_{\alpha=1}^{d} e_{\alpha}^{i} e_{\alpha}^{j} \frac{\partial h}{\partial y^{i}}(y, z) \frac{\partial h}{\partial y^{j}}(y, z) & =g^{i j}(y, z) \frac{\partial h}{\partial y^{i}}(y, z) \frac{\partial h}{\partial y^{j}}(y, z) \\
& =\left\|\operatorname{grad}_{L} h(y, z)\right\|_{g}^{2}
\end{aligned}
$$

for $\left(y, z,\left(e_{j}^{i}\right)\right)$ with $\widetilde{\varphi}^{-1}\left(y, z,\left(e_{j}^{i}\right)\right) \in O(\mathcal{L})$.

We can easily prove Theorem 2.8 .

Proof of TheOrem 2.8. Let $x$ be an element in $Q_{X}$. Recall that

$$
Y_{\lambda}^{x}(t)=M_{\lambda}^{x}(t)+\frac{h\left(X^{x}(\lambda t)\right)-h(x)}{\sqrt{\lambda}} .
$$

Since

$$
\sup _{t \geq 0} \frac{\left|h\left(X^{x}(\lambda t)\right)-h(x)\right|}{\sqrt{\lambda}} \leq \frac{2\|h\|_{\infty}}{\lambda} \rightarrow 0 \quad(\lambda \rightarrow \infty),
$$

we have only to show that $M_{\lambda}^{x} \rightarrow W_{\langle f\rangle(x)}$ in law as $\lambda \rightarrow \infty$. To this end it suffices to show that

$$
\left\langle M_{\lambda}^{x}\right\rangle(t) \rightarrow\left(\int_{M}\left\|\operatorname{grad}_{L} h\right\|_{g} d m_{x}\right) \cdot t \quad(\lambda \rightarrow \infty) \text { in probability }
$$

for $t \geq 0$ by [12, Corollary 1 and references therein]. Noticing that $x \in Q_{X}$, we obtain

$$
\begin{aligned}
\left\langle M_{\lambda}^{x}\right\rangle(t)=\frac{1}{\lambda} \int_{0}^{\lambda t} \| \operatorname{grad}_{L} h\left(X^{x}(s)\right) & \|_{g}^{2} d s=\left(\frac{1}{\lambda t} \int_{0}^{\lambda t}\left\|\operatorname{grad}_{L} h\left(X^{x}(s)\right)\right\|_{g}^{2} d s\right) \cdot t \\
& \rightarrow\left(\int_{M}\left\|\operatorname{grad}_{L} h\right\|_{g}^{2} d m_{x}\right) \cdot t \quad(\lambda \rightarrow \infty) P^{W} \text {-a.s. }
\end{aligned}
$$

This completes the proof of the theorem. 
REMARK 4.1. When $M$ is a mapping torus constructed by a topological dynamical system and $X$ is the leafwise Brownian motion induced by a natural leafiwise smooth Riemannian metric, the corresponding result to Theorem 2.8 is obtained in [14].

Next we consider the case when there is only one $A$-harmonic probability measure on $M$. Then we obtain the following.

LEMMA 4.2. The following are equivalent.

(i) An A-harmonic probability measure exists uniquely.

(ii) There exists an A-harmonic probability measure $m$ such that for any $f \in C(M)$, $(1 / t) \int_{0}^{t} T(s) f d$ s converges to $\int_{M} f d m$ uniformly as $t \rightarrow \infty$.

(iii) For any $f \in C(M)$, there exists a number $C(f)$ depending only on $f$ such that for any $x \in M,(1 / t) \int_{0}^{t} f\left(X^{x}(s)\right)$ ds converges to $C(f)$ in $L^{2}\left(P^{W}\right)$ as $t \rightarrow \infty$.

(iv) For any $f \in C(M)$, there exists a number $C(f)$ depending only on $f$ such that for any $x \in M,(1 / t) \int_{0}^{t} f\left(X^{x}(s)\right) d s$ converges to $C(f)$ in probability as $t \rightarrow \infty$.

(v) For any $f \in C(M)$, there exists a number $C(f)$ depending only on $f$ such that for any $x \in M,(1 / t) \int_{0}^{t} T(s) f(x) d s$ converges to $C(f)$ as $t \rightarrow \infty$.

Proof. ((i) $\Rightarrow$ (ii)). Let $m$ be an $A$-harmonic probability measure. Suppose that (ii) is false. Then there exist an element $f_{0}$ in $C(M)$, a positive number $\varepsilon_{0}$, a sequence $\left\{x_{j}\right\}$ of points in $M$ and a sequence of positive numbers $\left\{t_{j}\right\}$ such that $\lim _{j \rightarrow \infty} t_{j}=\infty$ and

$$
\left|\frac{1}{t_{j}} \int_{0}^{t_{j}} T(s) f_{0}\left(x_{j}\right) d s-\int_{M} f_{0} d m\right| \geq \varepsilon_{0} .
$$

Choosing a subsequence we may assume that the limit

$$
J(f)=\lim _{j \rightarrow \infty} \frac{1}{t_{j}} \int_{0}^{t_{j}} T(s) f\left(x_{j}\right) d s
$$

exists for any $f \in C(M)$. By the Riesz representation theorem, there exists a Borel probability measure $m^{\prime}$ such that $J(f)=\int_{M} f d m^{\prime}$ for each $f \in C(M)$. By substituting $T(t) f$ for $f$, it is easy to see that $m^{\prime}$ is an $A$-harmonic probability measure. The inequality (4.1) implies that $m \neq m^{\prime}$.

$$
\text { ((ii) } \Rightarrow \text { (iii)). Considering } f-C(f) \text {, we have only to show }
$$

$$
\lim _{t \rightarrow \infty} E\left[\left(\frac{1}{t} \int_{0}^{t} f\left(X^{x}(s)\right) d s\right)^{2}\right]=0
$$

whenever $(1 / t) \int_{0}^{t} T(s) f d s$ converges to 0 uniformly in $x$ as $t \rightarrow \infty$. For $t>0$ we have

$$
\begin{aligned}
I(t) & =E\left[\left(\frac{1}{t} \int_{0}^{t} f\left(X^{x}(s)\right) d s\right)^{2}\right] \\
& =\frac{2}{t^{2}} \int_{0}^{t} d r \int_{r}^{t} E\left[f\left(X^{x}(r)\right) f\left(X^{x}(s)\right)\right] d s \\
& =\frac{2}{t^{2}} \int_{0}^{t} d r \int_{r}^{t} E\left[f\left(X^{x}(r)\right)(T(s-r) f)\left(X^{x}(r)\right)\right] d s
\end{aligned}
$$




$$
\begin{aligned}
& =\frac{2}{t^{2}} \int_{0}^{t} d r \int_{0}^{t-r} E\left[f\left(X^{x}(r)\right)(T(s) f)\left(X^{x}(r)\right)\right] d s \\
& =\frac{2}{t^{2}} \int_{0}^{t} E\left[f\left(X^{x}(r)\right) \int_{0}^{t-r}(T(s) f)\left(X^{x}(r)\right) d s\right] d r \\
& =\frac{2}{t^{2}} \int_{0}^{t}(t-r) E\left[f\left(X^{x}(r)\right) \frac{1}{t-r} \int_{0}^{t-r}(T(s) f)\left(X^{x}(r)\right) d s\right] d r \\
& =2 \int_{0}^{1}(1-r) E\left[f\left(X^{x}(t r)\right) \frac{1}{t(1-r)} \int_{0}^{t(1-r)}(T(s) f)\left(X^{x}(t r)\right) d s\right] d r .
\end{aligned}
$$

Here the third equality follows from the Markov property of $X=\left\{X^{x}\right\}_{x \in M}$, the fourth equality is obtained by the change of variable $s-r \mapsto s$, the fifth equality is a consequence of the Fubini theorem, and the last equality is obtained by the change of variable $r \mapsto t r$. Thus we have

$$
|I(t)| \leq 2\|f\|_{\infty} \int_{0}^{1}(1-r)\left\|\frac{1}{t(1-r)} \int_{0}^{t(1-r)} T(s) f d s\right\|_{\infty} d r \rightarrow 0 \quad(t \rightarrow \infty)
$$

by the bounded convergence theorem. Hence we have (4.2).

(iii) $\Rightarrow$ (iv) and (iv) $\Rightarrow$ (v) hold immediately.

$((\mathrm{v}) \Rightarrow(\mathrm{i}))$. The ergodic theorem for $\{T(t)\}_{t \geq 0}$ yields that $C(f)=\int_{M} f d m$ holds for any $A$-harmonic probability measure $m$ (see [1, Theorem 7.3] and [4, Theorem VIII.7.1 and Theorem VIII.7.5]). Hence an $A$-harmonic probability measure exists uniquely.

REMARK 4.3. If $M$ is a mapping torus constructed by a topological dynamical system and $X$ is the leafwise Brownian motion, the unique ergodicity of the base dynamical system is also equivalent to the preceding (i)-(v). The proof of this fact is given in [14].

Now we state a version of Theorem 2.8 in the case when there is only one $A$-harmonic probability measure on $M$.

THEOREM 4.4. Assume that there exists a unique A-harmonic probability measure $m$. For a real-valued function $h \in C_{L}^{2}(M)$ let $f=A h$ and consider the process $Y_{\lambda}^{x}$ defined by (2.14). Then for any point $x \in M$, the processes $Y_{\lambda}^{x}$ converge in law to the Brownian motion $W_{\langle f\rangle}$ with variance $\langle f\rangle$ f for each time $t \geq 0$ as $\lambda \rightarrow \infty$, where

$$
\langle f\rangle=\int_{M}\left\|\operatorname{grad}_{L} h\right\|_{g}^{2} d m
$$

PROOF. We use the notation in the proof of Theorem 2.8. We need only to show that

$$
\left\langle M_{\lambda}^{x}\right\rangle(t) \rightarrow\left(\int_{M}\left\|\operatorname{grad}_{L} h\right\|_{g}^{2} d m\right) \cdot t \quad(\lambda \rightarrow \infty) \text { in probability }
$$

for any $x \in M$ and $t \geq 0$. From (iv) of Lemma 4.2, we have

$$
\left\langle M_{\lambda}^{x}\right\rangle(t)=\left(\frac{1}{\lambda t} \int_{0}^{\lambda t}\left\|\operatorname{grad}_{L} h\left(X^{x}(s)\right)\right\|_{g}^{2} d s\right) \cdot t \rightarrow\left(\int_{M}\left\|\operatorname{grad}_{L} h\right\|_{g}^{2} d m\right) \cdot t \quad(\lambda \rightarrow \infty)
$$

in probability for any $x \in M$ and $t \geq 0$. Now the proof of the theorem is complete. 
Finally we give two examples of uniquely ergodic $(1 / 2) \Delta_{g}$-leafwise diffusions, i.e., leafwise Brownian motions on foliated spaces.

EXAMPLE 4.5 (The mapping torus of a $\mathbb{Z}^{d}$-action on the $d$-torus). Let $\mathbb{T}^{d}=\mathbb{R}^{d} / \mathbb{Z}^{d}$ be the $d$-torus and $\left\{a_{1}, a_{2}, \ldots, a_{d}\right\}$ a set of real numbers such that $1, a_{1}, a_{2}, \ldots, a_{d}$ are linearly independent over $\mathbb{Q}$. Consider the $\mathbb{Z}^{d}$-action on $\mathbb{T}^{d}$ given by

$$
F^{n}: \mathbb{T}^{d} \ni\left(x_{1}, x_{2}, \ldots, x_{d}\right)+\mathbb{Z}^{d} \mapsto\left(x_{1}+n_{1} a_{1}, x_{2}+n_{2} a_{2}, \ldots, x_{d}+n_{d} a_{d}\right)+\mathbb{Z}^{d} \in \mathbb{T}^{d}
$$

for $n=\left(n_{1}, n_{2}, \ldots, n_{d}\right) \in \mathbb{Z}^{d}$. A $\mathbb{Z}^{d}$-action on $\mathbb{R}^{d} \times \mathbb{T}^{d}$ is also defined by $\mathbb{R}^{d} \times \mathbb{T}^{d} \ni$ $(u, x) \mapsto\left(u-n, F^{n} x\right) \in \mathbb{R}^{d} \times \mathbb{T}^{d}$ for $n \in \mathbb{Z}^{d}$. The quotient space $\left(\mathbb{R}^{d} \times \mathbb{T}^{d}\right) / \mathbb{Z}^{d}$ is a compact foliated space endowed with a natural leafwise smooth Riemannian metric $g$. The space is called the mapping torus of the action $F=\left\{F^{n}\right\}_{n \in \mathbb{Z}^{d}}$ and we denote it by $\mathbb{T}_{F}^{d}$. Each of the leaves of $\mathbb{T}_{F}^{d}$ is identified with $\mathbb{R}^{d}$. Let $B=\{B(t)\}_{t \geq 0}$ be a $d$-dimensional standard Brownian motion defined on a probability space $(\Omega, \mathcal{F}, P)$. The stochastic processes $X=\left\{X^{x}\right\}_{x \in \mathbb{T}_{F}^{d}}$ on $\mathbb{T}_{F}^{d}$ are defined so that $X^{\pi_{F}(u, x)}(t)=\pi_{F}(u+B(t), x)$ for $t \geq 0$, where $\pi_{F}: \mathbb{R}^{d} \times \mathbb{T}^{d} \rightarrow \mathbb{T}_{F}^{d}$ is the natural projection. It is easy to see that $X$ has the same distribution as the leafwise Brownian motion on $\mathbb{T}_{F}^{d}$. Moreover we can show that there is a one-to-one correspondence between the set of harmonic probability measures for $X$ on $\mathbb{T}_{F}^{d}$ and that of invariant probability measures for $F$ in the same way as [14]. The correspondence is explicit in the sence that if a harmonic probability measure $m_{\mu}$ for $X$ is correspondence to an invariant probability measure $\mu$ for $F$, we have

$$
\int_{\mathbb{T}_{F}^{d}} f d m_{\mu}=\int_{[0,1)^{d} \times \mathbb{T}^{d}}\left(f \circ \pi_{F}\right) d\left(l^{d} \times \mu\right)
$$

for any continuous function $f$ on $\mathbb{T}_{F}^{d}$, where $l^{d}$ is the $d$-dimensional Lebesgue measure on $[0,1)^{d}$. As is well-known, the normalized Haar measure $\mu_{H}$ is the only invariant probability measure for $F$. Therefore $m_{\mu_{H}}$ is a unique harmonic probability measure for $X$. Applying Theorem 4.4, if $(u, x)$ is an element in $\mathbb{R}^{d} \times \mathbb{T}^{d}$ and $f$ is of the form $f=(1 / 2) \Delta_{g} h$ with a function $h \in C_{L}^{2}\left(\mathbb{T}_{F}^{d}\right)$, then we see that

$$
\begin{aligned}
& \frac{1}{\sqrt{\lambda}} \int_{0}^{\lambda \cdot} f\left(X^{\pi_{F}(u, x)}(s)\right) d s \\
& \quad=\frac{1}{\sqrt{\lambda}} \int_{0}^{\lambda \cdot} f\left(\pi_{F}(u+B(s), x)\right) d s \rightarrow W_{\langle f\rangle}(\cdot) \quad(\lambda \rightarrow \infty) \text { in law },
\end{aligned}
$$

where $W_{\langle f\rangle}$ is the one-dimensional Brownian motion with variance

$$
\left(\int_{\mathbb{T}_{F}^{d}}\left\|\operatorname{grad}_{L} h\right\|_{g}^{2} d m_{\mu_{H}}\right) \cdot t=\left(\int_{[0,1)^{d} \times \mathbb{T}^{d}} \sum_{i=1}^{d}\left(\frac{\partial\left(h \circ \pi_{F}\right)}{\partial u^{i}}\right)^{2} d\left(l^{d} \times \mu_{H}\right)\right) \cdot t
$$

for each time $t \geq 0$.

EXAMPLE 4.6 (The stable foliation of the geodesic flow on a compact Riemannian manifold of negative curvature). Let $M$ be a compact smooth Riemannian manifold of negative 
sectional curvature, $S M$ the unit tangent bundle to $M,\left\{\phi_{t}\right\}_{t \in \mathbb{R}}$ the geodesic flow on $S M$ and $W^{s}(v)$ the stable manifold of the element $v$ in $S M$, i.e.,

$$
W^{s}(v)=\left\{w \in S M: \text { there exists } s \in \mathbb{R} \text { such that } \lim _{t \rightarrow \infty} d\left(\phi_{t+s} v, \phi_{t} w\right)=0\right\} .
$$

It is well known that $S M$ is a compact foliated space with $W^{s}(v)$ as the leaf passing through $v \in S M$. The Riemannian metric on $M$ induces a leafwise smooth Riemannian metric $g$ on $S M$. Hence the leafwise Laplace-Beltrami operator $\Delta_{g}$ and the leafwise Brownian motion $X=\left\{X^{v}\right\}_{v \in S M}$ are defined on $S M$. It should be noted that Ledrappier discusses about such a process (see [10] and [11]). In particular he showed that a harmonic probability measure $m$ for $X$ exists uniquely in [11]. Therefore, applying Theorem 4.4, we see that

$$
\frac{1}{\sqrt{\lambda}} \int_{0}^{\lambda \cdot} f\left(X^{v}(s)\right) d s \rightarrow W_{\langle f\rangle}(\cdot) \quad(\lambda \rightarrow \infty) \text { in law }
$$

whenever $v$ is an element in $S M, f$ is of the form $f=(1 / 2) \Delta_{g} h$ with a function $h \in$ $C_{L}^{2}(S M)$ and $W_{\langle f\rangle}$ is the Brownian motion with variance

$$
\left(\int_{S M}\left\|\operatorname{grad}_{L} h\right\|_{g}^{2} d m\right) \cdot t
$$

for each time $t \geq 0$.

Acknowledgment. The author would like to express his gratitude to Professor Takehiko Morita for valuable comments and advice. The author would also like to thank anonymous referee for helpful comments and suggestions.

\section{REFERENCES}

[ 1] A. CAndel, The harmonic measures of Lucy Garnett, Adv. Math. 176 (2003), 187-247.

[2] A. Candel and L. Conlon, Foliations I, Amer. Math. Soc., Providence, 2000.

[3] A. Candel And L. Conlon, Foliations II, Amer. Math. Soc., Providence, 2003.

[ 4 ] N. Dunford and J. T. Schwartz, Linear Operators Part I, John Wiley \& Sons, New York, 1988.

[ 5 ] L. GARnEtT, Foliations, the ergodic theorem and Brownian motion, J. Funct. Anal. 51 (1983), $285-311$.

[6] N. IKeda And S. Watanabe, Stochastic differential equations and diffusion processes 2nd. ed., North Holland, Amsterdam, 1989.

[ 7 ] M. KANAI, A new approach to the rigidity of discrete group actions, Geom. Funct. Anal. 6 (1996), 943-1056.

[ 8 ] I. Karatzas AND S. E. Shreve, Brownian motion and stochastic calculus 2nd. ed., Springer-Verlag, New York, 1991.

[9] C. Kipnis and S. R. S. Varadhan, Central limit theorem for additive functionals of reversible Markov processes and applications to simple exclusions, Comm. Math. Phys. 104 (1986), 1-19.

[10] F. Ledrappier, Central limit theorem in negative curvature, Ann. Probab. 23 (1995), 1219-1233.

[11] F. LEDRAPPIER, Ergodic properties of the stable foliations, Ergodic theory and related topics III (Güstrow, 1990), 131-145, Lecture Notes in Math. 1514, Springer, Berlin, 1992.

[12] R. SH. LiptSER AND A. N. ShiRYAEV, On a problem of necessary and sufficient conditions in the functional central limit theorem for local martingales, Z. Wahrsch. Verw. Gebiete 59 (1982), 311-318.

[13] C. C. Moore And C. L. Schochet, Global analysis on foliated spaces 2nd. ed., Cambridge University Press, New York, 2006. 
[14] T. Morita AND K. SUZAKI, Harmonic measures for leafwise Brownian motions on mapping tori, Preprint 2012.

[15] Y. OCHI, Limit theorems for a class of diffusion processes, Stochastics 15 (1985), 251-269.

[16] L. C. G. Rogers And D. Williams, Diffusions, Markov processes, and martingales Vol. 2. Itô calculus, Cambridge University Press, Cambridge, 2000.

[17] P. WALCZAK, Dynamics of foliations, groups and pseudogroups, Birkhäuser Verlag, Basel, 2004.

DEPARTMENT OF MATHEMATICS

Graduate SCHOOL OF SCIENCE

OSAKA UNIVERSITY

TOYONAKA OSAKA 560-0043

JAPAN

E-mail address: k-suzaki@cr.math.sci.osaka-u.ac.jp 